\title{
Photospheric downward plasma motions in the quiet Sun ${ }^{\star}$
}

\author{
C. Quintero Noda ${ }^{1,2}$, B. Ruiz Cobo ${ }^{1,2}$, and D. Orozco Suárez ${ }^{1,2}$ \\ 1 Instituto de Astrofísica de Canarias, 38200 La Laguna, Tenerife, Spain \\ e-mail: cqn@iac.es \\ 2 Departamento de Astrofísica, Univ. de La Laguna, 38205 La Laguna, Tenerife, Spain
}

Received 19 January 2014 / Accepted 12 May 2014

\begin{abstract}
Context. We analyze spectropolarimetric data taken with the Hinode spacecraft in quiet solar regions at the disk center. Distorted redshifted Stokes $V$ profiles are found that show a characteristic evolution that always follows the same sequence of phases.

Aims. We aim to characterize the statistical properties of these events and recover the stratification of the relevant physical quantities to understand the nature of the mechanism behind them.

Methods. We studied the statistical properties of these events using spectropolarimetric data from Hinode/SP. We also examined the upper photosphere and the low chromosphere using $\mathrm{Mg} \mathrm{I} \mathrm{b}_{2}$ and $\mathrm{Ca}$ II $\mathrm{H}$ data from Hinode. Finally, we applied the SIRGAUSS inversion code to the polarimetric data to infer the atmospheric stratification of the physical parameters. We also obtained these physical parameters taking into account dynamical terms in the equation of motion.

Results. The Stokes $V$ profiles display a bump that evolves in four different time steps, and the total process lasts 108 seconds. The Stokes $I$ shows a strongly bent red wing and the continuum signal exhibits a bright point inside an intergranular lane. This bright point is correlated with a strong redshift in the $\mathrm{Mg} \mathrm{I} \mathrm{b}_{2}$ line and a bright feature in $\mathrm{Ca}$ II $\mathrm{H}$ images. The model obtained from the inversion of the Stokes profiles is hotter than the average quiet-Sun model, with a vertical magnetic field configuration and field strengths in the range of $\mathrm{kG}$ values. It also presents a line of sight velocity stratification with a Gaussian perturbation, the center of which is moving to deeper layers with time. The Gaussian perturbation is also found in the gas pressure and density stratification obtained taking into account dynamical terms in the equation of motion.

Conclusions. We have examined a particular type of event that can be described as a plasmoid of hot plasma that is moving downward from the top of the photosphere, placed over intergranular lanes and always related to strong magnetic field concentrations. We argue that the origin of this plasmoid might be magnetic reconnection that is taking place in the chromosphere.
\end{abstract}

Key words. Sun: granulation - Sun: photosphere - Sun: magnetic fields

\section{Introduction}

Understanding of quiet-Sun magnetism has improved enormously in recent years, mainly thanks to the successful performance of several instruments operating beyond the Earth's atmosphere, such as the spectropolarimeter (Lites et al. 2013) onboard the Hinode satellite (Kosugi et al. 2007; Tsuneta et al. 2008) and IMaX (Martínez Pillet et al. 2011a) onboard Sunrise (Barthol et al. 2011; Solanki et al. 2010). These instruments have provided data with spatial resolutions similar to, or better than, those available in the past, see, for example, SST (Scharmer et al. 2003), and very significantly improved temporal consistency, going beyond the typical granular evolutionary time scales. One of the most interesting features in the quiet-Sun photosphere is the high-speed magnetized flows that can be deduced from highly distorted Stokes $V$ profiles detected in different solar regions (Shimizu et al. 2008). These profiles present a strongly redshifted signal and a multiple lobe structure. However, in spite of the efforts of previous authors, the origin of these strong flows and their length scale in the solar atmosphere is still debated.

One of the proposed responsible mechanisms is a convective instability inside a magnetic flux tube. The convective collapse process was first suggested on theoretical grounds by Parker (1978), Webb \& Roberts (1978), and Spruit (1979). Recent

\footnotetext{
* Appendix A is available in electronic form at http://www. aanda.org
}

studies have tentatively identified this mechanism through observations (Bellot Rubio et al. 2001; Nagata et al. 2008; Fischer et al. 2009) as the amplification of the magnetic field induced by a strong downflow, with line-of-sight (LOS) component speeds of $6 \mathrm{~km} \mathrm{~s}^{-1}$. It has also been proposed that these downflows hit dense layers and rebound as supersonic upflows (Grossmann-Doerth et al. 1998). These upflows would explain the extremely blueshifted signals observed by Socas-Navarro \& Manso Sainz (2005). Another possible mechanism that could produce the strong flows is magnetic reconnection (Parker 1963). This mechanism was proposed by Borrero et al. (2013) to explain the photospheric stratification obtained through the inversion of the strongly Doppler-shifted profiles first reported in Borrero et al. (2010).

Chromospheric layers also present ubiquitous high-speed plasma events in the form of needle-like structures called spicules that appear in limb observations (Beckers 1968). The recent rediscovery of a new type of spicule (type II spicules, de Pontieu et al. 2007) with shorter lifetimes (10-150 s), smaller diameters $(<200 \mathrm{~km}$ compared to $<500 \mathrm{~km}$ for type I spicules), and faster rise times confirms the complex nature of these solar structures, which are still far from being well understood. The solar disk-center counterpart of the limb spicules was first studied by Langangen et al. (2008) and then by Sekse et al. (2012,2013), who revealed highly blueshifted signals in the chromospheric $\mathrm{Ca}$ II line, named by the first author rapid blueshifted excursions (RBEs). They are characterized by a 
length of about $1.2 \mathrm{Mm}$ and a width of $0.5 \mathrm{Mm}$. The RBEs appear to be located near strong magnetic concentrations (network patches), but not on top of them. The Doppler shifts associated with the RBEs suggest velocities of $15-20 \mathrm{~km} \mathrm{~s}^{-1}$. Owing to the highly energetic nature of RBEs, previous authors concluded that the asymmetric blueward spectral profile has to be generated by a magnetic reconnection process that occurs in the chromosphere.

In this paper we examine the polarimetric properties of Fe I Stokes $V$ profiles from Hinode/SP that display a strong Doppler-shifted signal on the red side of the line. We then follow their vertical trace through an analysis of the upper photospheric $\mathrm{Mg} \mathrm{I}_{2}$ line and chromospheric Ca II H spectral band. Finally, we infer the atmospheric stratification of the physical parameters using the SIRGAUSS inversion code. The aim of the study is to reveal the nature of these strong Doppler-shifted signals and the physical mechanism that produces them.

\section{Observations and data analysis}

The polarimetric data used in this paper were obtained with the spectropolarimeter (SP) onboard the Hinode satellite. In particular, we analyzed different data sets: one consisting of two scans, also called normal maps, of 328" $\times 154^{\prime \prime}$ recorded on 2007 March 10 and 2007 October 15 that point at the disk center. The SP instrument measures the Stokes spectra of the Fe I 6301.5 and $6302.5 \AA$ lines with a spectral sampling of $2.15 \mathrm{pm} \mathrm{pixel}^{-1}$ and a spatial sampling of $0.16^{\prime \prime}$. The exposure time is $4.8 \mathrm{~s}$ per slit position, which makes it possible to achieve a noise level of $1.1 \times 10^{-3} I_{\mathrm{c}}$ in Stokes $V$ and $1.2 \times 10^{-3} I_{\mathrm{c}}$ in Stokes $Q$ and $U$, where $I_{\mathrm{c}}$ stands for the continuum intensity.

The second data set is a time-series observation based on a raster scan of 18 scanning steps with $1.6 \mathrm{~s}$ of exposure time per slit position, spanning three hours of observation on 2007 September 25. In this case, the field of view (FOV) is $2.9^{\prime \prime} \times 38^{\prime \prime}$ and the final time cadence is $36 \mathrm{~s}$. These observations belong to the Hinode Operation Plan 14, entitled Hinode/Canary Islands campaign, and allow us to analyze the evolution of small magnetic structures in the quiet Sun, although the noise level is poorer than that of the first data set.

The Hinode Broadband Filter Imager (BFI; Tsuneta et al. 2008) instrument acquired simultaneous images of the photosphere in the $\mathrm{CN}$ band head at $388.3 \mathrm{~nm}$ (filter width of $0.52 \mathrm{~nm}$ ) and of the chromosphere in the Ca II H line at $396.85 \mathrm{~nm}$ (filter width of $0.22 \mathrm{~nm}$ ). The exposure times were $0.1 \mathrm{~s}$ and $0.3 \mathrm{~s}$. The BFI covered a region of $19.12^{\prime \prime} \times 74.1^{\prime \prime}$ with a pixel size of $0.055^{\prime \prime}$ and took images with a cadence of $30 \mathrm{~s}$.

We also have data from the Narrowband Filter Imager (NFI) consisting of shutterless Stokes $I$ and $V$ images in the red and blue wings of the $\mathrm{Mg} \mathrm{I} \mathrm{b}_{2}$ line at $517.27 \mathrm{~nm}$. From these data we constructed magnetograms and Dopplergrams as

$M=\frac{1}{2}\left(\frac{V_{\mathrm{b}}}{I_{\mathrm{b}}}-\frac{V_{\mathrm{r}}}{I_{\mathrm{r}}}\right)$

$D=\frac{I_{\mathrm{b}}-I_{\mathrm{r}}}{I_{\mathrm{b}}+I_{\mathrm{r}}}$

where $r$ and $b$ indexes stand for the red and blue sides of the line.

Data from $\mathrm{CN}$ and $\mathrm{Ca}$ II $\mathrm{H}$ spectral bands are a series of images of the same FOV, taken every $30 \mathrm{~s}$. Because of the difference between the cadence of the $\mathrm{CN}$ and $\mathrm{Ca}$ II $\mathrm{H}$ images and the cadence of each Hinode/SP map (36 s), we chose the image of the $\mathrm{CN}$ and $\mathrm{Ca}$ II $\mathrm{H}$ bands that was closest in time to the SP map snapshot. We selected the time reference from the central slit of the SP map (slit 9) and chose the image of $\mathrm{CN}$ or $\mathrm{Ca}$ II $\mathrm{H}$ with the observing time closest to the ninth slit time. The broad-band images we chose come before the slit acquisition time or after it, but the temporal difference between them was never longer than $18 \mathrm{~s}$, which corresponds to half the time cadence of each Hinode/SP map.

For the $\mathrm{Mg} \mathrm{I} \mathrm{b}_{2}$ data we followed the same method as for the $\mathrm{Ca}$ II $\mathrm{H}$ images, but in this case the FOV of the $\mathrm{Mg} \mathrm{I} \mathrm{b}_{2}$ is larger than the FOV of the Hinode/SP maps. We selected the region of the magnesium image that corresponds to the Hinode/SP raster scan map to analyze the possible relationship between them using strong circular polarization signals on the $\mathrm{Mg} \mathrm{I} \mathrm{b}_{2}$ magnetograms as reference.

The events of interest in this study are those representing a strong Doppler-shifted Stokes $V$ signal. We followed the method presented by Martínez Pillet et al. (2011b) to find them, examining the Stokes $V$ signal at $\pm 272 \mathrm{~m} \AA$ from the center of the Fe I $6302.5 \AA$ line and generating far-wing magnetograms from the red and the blue sides of that line. We then analyzed these magnetograms looking for pixels with a Stokes $V$ signal higher than $0.5 \%$ of the mean continuum intensity of the map (hereafter $I_{\mathrm{c}}$ ). As the detected events were grouped in patches of different sizes, we decided to define a minimum area of detection of four pixels to avoid contribution of isolated bad pixels.

We found many more redshifted than blueshifted patches. We detected a family of events that show a characteristic evolution of their Stokes $V$ profile shapes inside these redshifted cases. We analyzed their evolution using the raster scan observing mode, studying at the same time the information available from higher layers where the $\mathrm{Mg} \mathrm{I} \mathrm{b}_{2}$ and $\mathrm{Ca}$ II $\mathrm{H}$ lines are formed.

Finally, the atmospheric models of these events were inferred using the SIRGAUSS inversion code (Bellot Rubio 2003). This inversion code is a modification of the code Stokes inversion based on response functions (SIR; Ruiz Cobo \& del Toro Iniesta 1992) and considers Gaussian perturbations along the LOS stratification for the atmospheric parameters.

\section{General properties of the redshifted events}

\subsection{Polarimetric characteristics and evolution}

The detected redshifted events always appear at intergranular lanes. The Stokes profiles change their shape, as Fig. 1 shows. Each column corresponds to the evolution of the Stokes profiles from a single pixel in one event. The temporal cadence is $36 \mathrm{~s}$. We have marked the most important part of the event that starts at $\Delta t=0$. Before the beginning of the process, Stokes $V$ is strongly redshifted and, in addition, presents a continuum enhancement in Stokes $I$ that starts at $\Delta t=-72$. The Stokes $V$ profile then suddenly changes its shape (purple; $\Delta t=0$ ); a small bump appears at the top part of the profile at the zero-crossing point (blue; $\Delta t=36$ ); then, it shifts to the red wing, distorting the Stokes $V$ red lobe (green; $\Delta t=72$ ); and finally it appears at the red wing of the Stokes $V$ profile (orange; $\Delta t=108$ ). After that instant, the Stokes $I$ continuum signal relaxes to values closer to those displayed by quiet-Sun intergralunar lanes, and the Stokes $V$ profiles present an extended red wing and evolve in two different ways: the profiles recover a typical antisymmetric Stokes $V$ shape without signal or, the most common behavior, the polarization signals vanish.

The Stokes $V$ profiles show a strongly redshifted signal during the whole process. Although the cores of Stokes $I$ (first column of Fig. 1) are not highly redshifted, the red wings are 

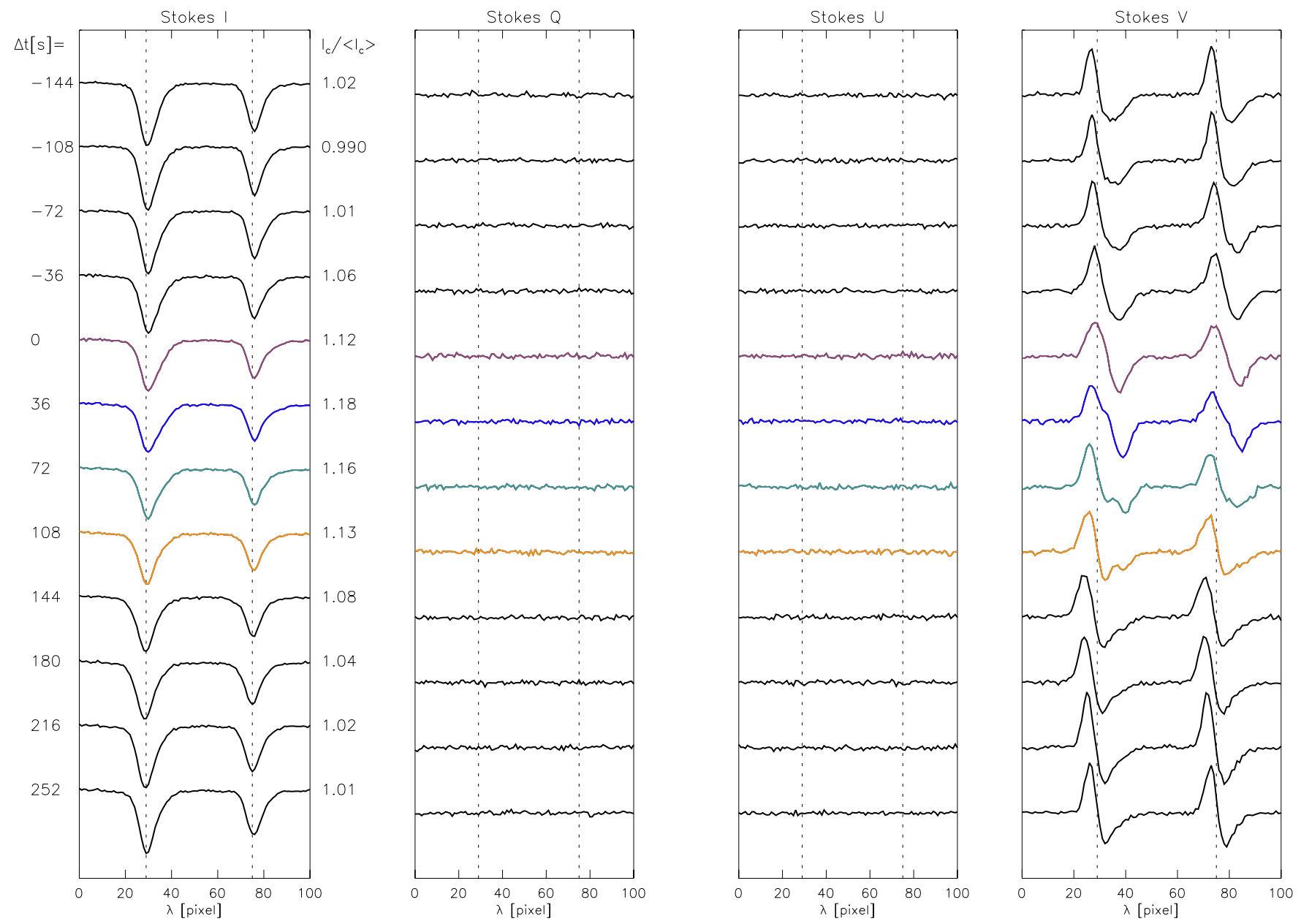

Fig. 1. Temporal evolution of a single pixel from one of the 11 detected cases in the SP time series. Each column corresponds to a different Stokes parameter, and time runs from top to bottom. Stokes $Q$ and $U$ amplitudes are always lower than the noise. Stokes $V$ shows a bump at the top-center of the line (purple; $\Delta t=0$ ) that apparently moves, crossing the center of the line to the red wing (orange; $\Delta t=108$ ), and finally vanishes. The appearance of the bump coincides with an enhancement of the continuum intensity signal (values at the right of the first column represent the continuum signal divided by the mean continuum intensity of the whole map). After the disappearance of the bump, the continuum intensity decreases.

strongly distorted. The Doppler shift and the continuum enhancement mentioned before start to decrease during the late steps (green and orange). Remarkably, the Stokes $V$ amplitude of the Fe I $6301.5 \AA$ line is always higher than the amplitude of $6302.5 \AA$, its neighboring Fe I line. This is an unexpected attribute of the Stokes $V$ profiles of these events because in the weak-field regime the first line, Fe I $6301.5 \AA$ (the least sensitive to the magnetic field line) always has a lower Stokes $V$ amplitude. In addition, although the wavelength location of the bump inside each of the Stokes $V$ profiles is the same for both lines, the bump is more prominent in the first line, that is, in Fe I 6301.5 $\AA$.

In the region where the event occurs, Stokes $V$ amplitudes are usually between $5 \%$ and $10 \%$ of the mean continuum signal $\left(I_{\mathrm{c}}\right)$, whereas $Q$ and $U$ amplitudes are always lower than the noise level (see Fig. 1), indicating that the transverse component of the magnetic field has to be small, which means that the magnetic structure is nearly vertical. Moreover, neither structures presenting opposite polarities nor linear polarization signals appear to be close to the region where the event evolves.

It is important to note for Stokes $I$ that the event appears in an intergranular lane, where continuum intensities in the quiet Sun have values in the range $0.8-0.95 I_{\mathrm{c}}$, while the pixels that display strong Stokes $V$ redshifted signals have Stokes $I$ continuum intensity values higher than $1.1 I_{\mathrm{c}}$. For this reason, this process always comes into view as a bright point in the continuum map. In addition, if we define the line depth of Stokes $I$ as the difference between the intensity of the continuum and the core of the line, we find that the Stokes I profiles have a small line depth in both lines, reaching values in the range $0.4-0.5 I_{\mathrm{c}}$, while typical quiet-Sun profiles usually present values of $0.7-0.8 I_{\mathrm{c}}$. This low line depth value is due to a significant increase of the line core intensity that exceeds the enhancement of the continuum signal.

\subsection{Lifetime, size, and rate of occurrence}

We detected 11 cases that show the same behavior as the one presented in Fig. 1 during the three hours that the time series lasts. A closer examination of their evolution allows determining a mean life time of $360 \pm 74 \mathrm{~s}$. To calculate the lifetime of each event we assumed that the initial stage corresponds to the simultaneous detection of the strongly redshifted signal in Stokes $V$ and the enhancement of the continuum Stokes I signal, while the last stage corresponds to the disappearance of the strongly redshifted Stokes $V$ signal and the relaxation of the Stokes $I$ continuum signal. The different positions of the bump displayed by the Stokes $V$ profile are indicated by colors in Fig. 1. We always 

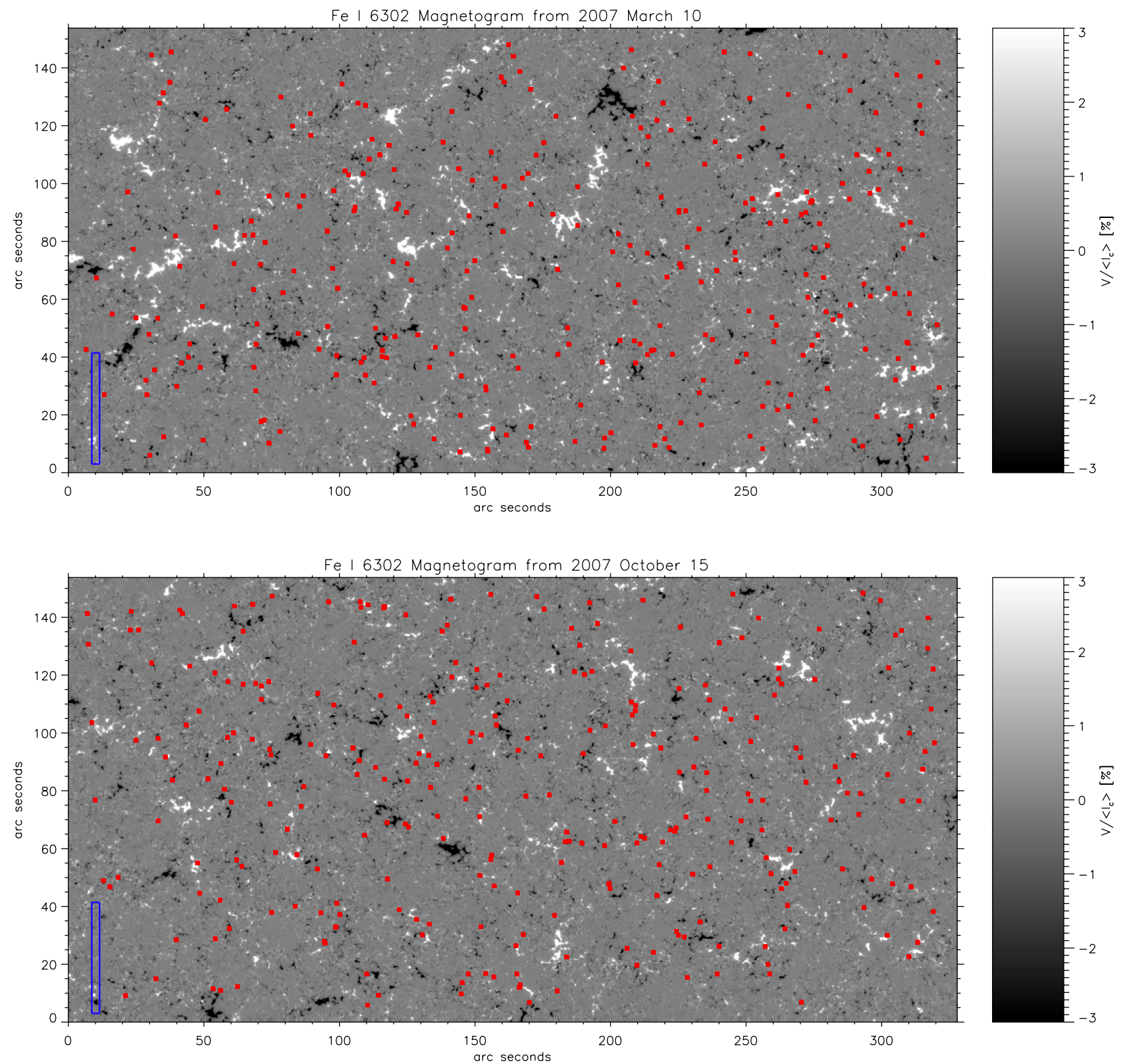

Fig. 2. Location of the redshifted events (red filled squares) in the Fe I $6302.5 \AA$ magnetogram from the normal maps recorded on 2007 March 10 (upper panel) and 2007 October 15 (bottom panel). The size of the filled squares is larger than the size of the events to make them visible. The left blue rectangle represents the FOV of the raster scan mode.

detected a different bump step in consecutive images, which indicates that the lifetime of each step has to be shorter than the cadence of the observing mode, that is, $36 \mathrm{~s}$. When we examined the Stokes $V$ signals from adjacent pixels inside the event for a single snapshot, we found the coexistence of one or two different and consecutive bump stages, that is, different colors in Fig. 1. This means that more than one bump step can be found inside the magnetized patch with a mean surface of $4 \pm 1.4$ pixels. Moreover, the events do not stay in the same place but move horizontally inside a $0.5 \operatorname{arcsec}^{2}$ area.

To establish a rate of occurrence, we examined the large FOV of the normal maps. We looked for profiles that present the same polarimetric characteristics as explained in the previous section. To analyze this enormous amount of data, we plotted a $5 \times 5$ pixel panel centered on the cases that show a strongly redshifted signal at $272 \mathrm{~m} \AA$ from the Stokes $V$ center. After visual inspection of each panel, we selected the events that have a spatial distribution similar to the cases examined using the timeseries observation. The total number of detected events was 302 for the map recorded on 2007 March 10 and 290 for the map of 2007 October 15. The continuum signal reveals that these events, as in the time series, appear as bright features in intergranular lanes. Concerning the distribution of these profiles with respect to the magnetic field topology, Fig. 2 shows the locations of the center of each event by red filled squares over the magnetogram maps obtained from the Fe I $6302.5 \AA$ line for both dates. The distribution of the events reveals that they are located in the surroundings of supergranular cells related to the strong magnetic activity represented by the network regions. However, the events never appear on top of these network regions.

The polarimetric analysis of the previous section revealed that the different bump phases, represented by colors in Fig. 1, last more than $100 \mathrm{~s}$. This lifetime is much longer than the exposure time of each slit position used to obtain the maps of Fig. 2, 
C. Quintero Noda et al.: Photospheric downward plasma motions in the quiet Sun
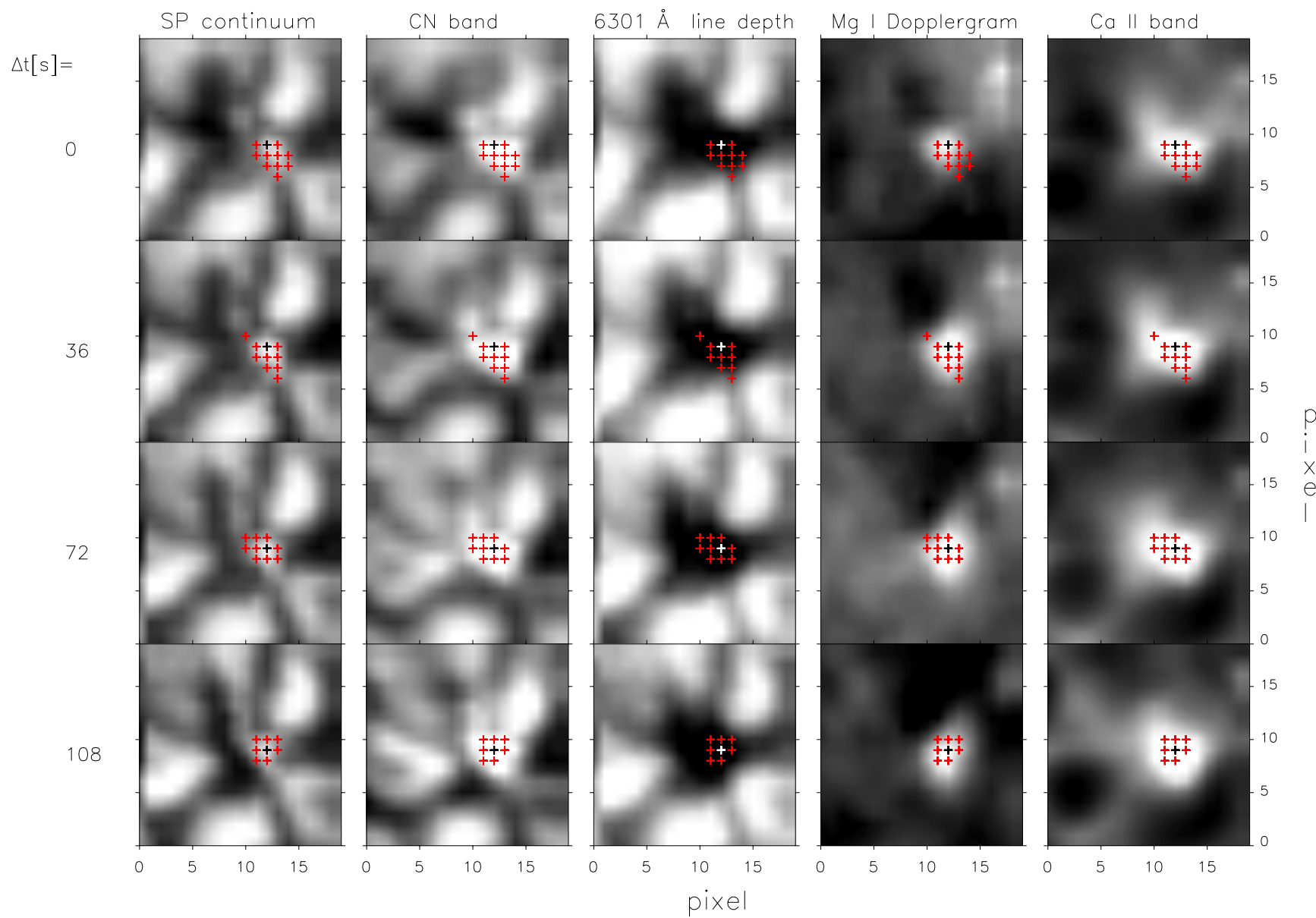

Fig. 3. Hinode/SP continuum signal (first column), CN spectral band (second column), line depth of the Fe I $6301.5 \AA$ A line (third column), Mg I $\mathrm{b}_{2}$ Dopplergram signal (with downflows in white and upflows in black) (fourth column), and Ca II H intensity maps (last column). Red crosses correspond to the locations of the pixels that are highly redshifted. The time interval runs from top to bottom and is the same as depicted in color in Fig. 1. The pixel analyzed in that figure is marked with a black cross (white in the third column). We have reduced the spatial resolution of CN, $\mathrm{Ca}$ II $\mathrm{H}$ and $\mathrm{Mg}$ I $\mathrm{b}_{2}$ images to match the Hinode/SP spatial resolution.

which was $4.8 \mathrm{~s}$, allowing us to consider the magnetograms as single snapshots. To find the rate of occurrence of these events we divided the mean number of cases detected in both maps $(296 \pm 8.4)$ by the area of one map $\left(328^{\prime \prime} \times 154^{\prime \prime}\right)$. The resulting rate of occurrence of $5.9 \times 10^{-3}$ cases per $\operatorname{arcsec}^{2}$ indicates that we would find nearly 60 different events in a FOV of $100 \times$ $100 \operatorname{arcsec}^{2}$. If we compare these properties with a typical granule structure (size of $1.5^{\prime \prime}$ and lifetime of $10 \mathrm{~min}$ ), we can establish that these events are an uncommon, small, and transient phenomenon that occurs inside of intergranular lane regions.

\subsection{Chromospheric response}

Figure 3 shows the $\mathrm{Ca}$ II $\mathrm{H}, \mathrm{CN}$, and $\mathrm{Mg} \mathrm{I}_{2}$ data. Time runs from top to bottom. The first column is the SP continuum signal. The second column is the $\mathrm{CN}$ spectral band. The third column is the line depth of the Fe I $6301.5 \AA$ line. The fourth column displays the $\mathrm{Mg} \mathrm{I} \mathrm{b}_{2}$ Dopplergram, and the fifth column shows the Ca II H images. We interpolated these maps to the SP spatial resolution to compare the $\mathrm{CN}, \mathrm{Ca}$ II $\mathrm{H}$ and $\mathrm{Mg} \mathrm{I}_{2}$ images with the Hinode/SP observations. Each row corresponds to a different time, running from top to bottom. We chose the same time steps we plotted in different colors in Fig. 1 starting at $\Delta t=0$. Red crosses indicate the position of the pixels of the selected event, that is, pixels with a strong enough signal at $272 \mathrm{~m} \AA$. Additionally, we marked with a black (or white in the third column) cross in each image the individual pixel displayed in Fig. 1.

The continuum map shows that the event always appears over a bright region inside an intergranular lane. The $\mathrm{CN}$ band presents the same properties, displaying a bright feature at the positions marked by the red crosses. The next column shows the line depth; a black patch means that the line depth is lower than $0.5 I_{\mathrm{c}}$, whereas a white patch indicates that the line depth is higher than $0.8 I_{\mathrm{c}}$. The event always occurs inside a black region, meaning that the line depth is very low during the process. As we mentioned in Sect. 3.1, the enhancement of the line core of the $6301.5 \AA$ is strong enough to produce a low Stokes $I$ line depth even when the continuum signal is higher than $1.1 I_{\mathrm{c}}$. This strong Stokes $I$ core enhancement complements the information of the previous section, where we noted the larger Stokes $V$ amplitude of this line, and indicates that the process forms in the upper photosphere.

Magnesium Dopplergrams show a strong downflow patch in the same region as where the event is detected. This patch with large downflows starts before, or at the same time as, the detection of the events by SP lines for the 11 different cases detected. We have omitted the magnesium magnetogram because it shows the same information as the Fe I $6302.5 \AA$ magnetogram: a unipolar patch isolated from opposite polarity regions. Finally, 


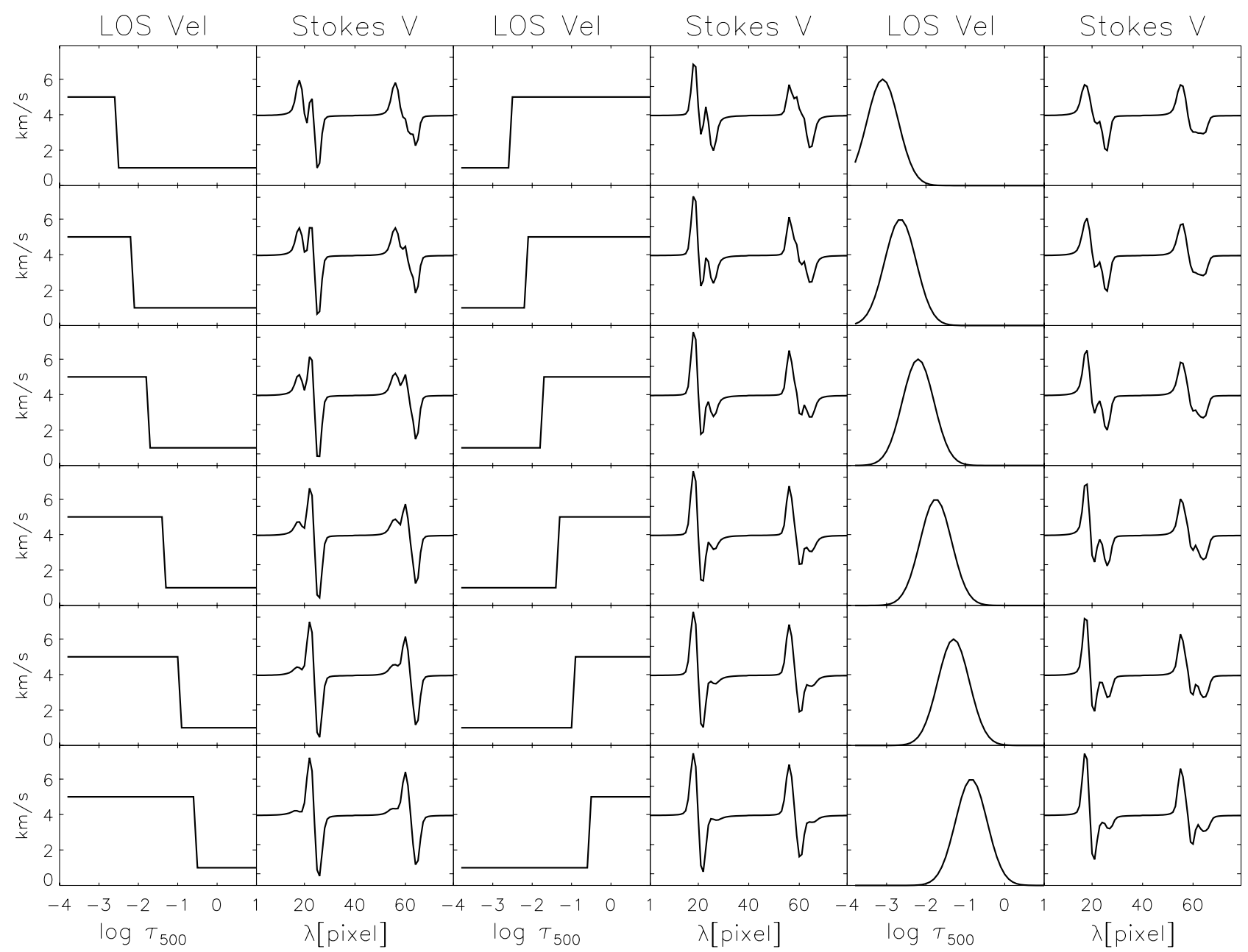

Fig. 4. Each pair of columns shows the LOS velocity stratification and the corresponding synthesized Stokes $V$ profiles. The temperature of the model used in the synthesis is hotter than the HSRA reference atmosphere. The magnetic field vector has a constant $1 \mathrm{kG}$ intensity and zero degrees in inclination and azimuth. The first column shows a LOS velocity jump of $5 \mathrm{~km} \mathrm{~s}^{-1}$ that moves from the top to the bottom of the photospheric layers. The second column shows the resulting Stokes $V$ profiles. The third column shows an opposite jump, with higher velocities at lower heights. The Stokes $V$ profiles obtained from this velocity configuration are plotted in the fourth column. The last column shows the synthetic profiles when the LOS velocity stratification has a Gaussian perturbation, as shown in the fifth column.

the $\mathrm{Ca}$ II $\mathrm{H}$ images show a bright feature where the pixels of the event are plotted, suggesting that these Fe I distorted profiles have a counterpart in the formation region of the $\mathrm{Ca}$ II $\mathrm{H}$ line.

The correlation between photospheric downflows and bright features in the $\mathrm{Ca}$ II $\mathrm{H}$ line has been reported before by Shimizu et al. (2008) and Fischer et al. (2009). The latter work revealed an extensive variation in the $\mathrm{Ca}$ II $\mathrm{H}$ signal during the evolution of the process. We also found this strong variation in the region where the event occurs. Because of this substantial intensity fluctuation and the small FOV of the time-series, we have no clear indication to establish whether the bright $\mathrm{Ca}$ II $\mathrm{H}$ feature was there before the detection of the event by the iron lines or whether it started at the same time. We can establish only that all the cases show a correlated bright point in $\mathrm{Ca}$ II $\mathrm{H}$ data and that this bright point never appears after the detection of the bump in the iron lines.

\section{Forward-modeling}

We can obtain physical information on the mechanism that produces these events by inverting the Stokes profiles using the SIR code. Because of the high freedom in modeling of the atmosphere allowed by the code it is advisable, to build a model whose profiles qualitatively show the same distortion as the observed ones before starting with the inversion of the Stokes profiles.

After many tests, we concluded that the bumps displayed by the observed Stokes $V$ profiles can be well reproduced using a model atmosphere that contains a strong jump in its LOS velocity stratification. However, the perturbation bump is not static in one position on the Stokes $V$ profiles when time evolves: it appears in the middle of the line, in the red lobe, and finally in the red wing. For this reason the synthetic profiles need different jump stratifications to reproduce the different locations of this bump.

Figure 4 shows different examples of the Stokes $V$ profiles that can be obtained using an ideal jump or a Gaussian profile in the LOS velocity stratification. The figure is divided into columns, with the LOS velocity stratification first and then the synthesized Stokes $V$ profile.

The amplitude of the Stokes $V$ profile for the Fe I $6301.5 \AA$ line is always lower than that of the second line when we use the temperature stratification from a quiet-Sun model such as the 
Harvard-Smithsonian Reference Atmosphere (HSRA; Gingerich et al. 1971). The easiest way we found to change the Stokes $V$ amplitude behavior was to choose an atmospheric model hotter than that given by the HSRA reference atmosphere model. The difference between temperature stratifications is larger in the region of line formation. With this atmospheric configuration we were able to reproduce the observed amplitude of Fe I $6301.5 \AA$ during the whole process. This hotter atmospheric model also reproduces the increase in the signal level of the line core observed in the previous section. For the magnetic field configuration we chose a vertical and constant magnetic field. We selected a constant field intensity through the atmosphere owing to the absence of significant area asymmetry in the Stokes $V$ profiles, and a vertical magnetic inclination because the Stokes $Q$ and $U$ signals are always below the noise.

The first column of Fig. 4 shows the first LOS velocity configuration, a jump of $5 \mathrm{~km} \mathrm{~s}^{-1}$ from top to bottom of the atmosphere, while the second column shows the synthetic and peculiar Stokes $V$ profiles that this velocity stratification produces. We discarded this solution because it produces a bump in the blue lobe, which is never recorded in the observational data.

The fourth column shows the Stokes $V$ profiles coming from a LOS velocity configuration with a jump of $5 \mathrm{~km} \mathrm{~s}^{-1}$ from the bottom to the top of the atmosphere, that is, from a LOS velocity component that is only present at the base of the photosphere. In this case, the Stokes $V$ profiles resemble the observed ones, and the evolution of the bump is reproduced as the jump goes through different heights. However, in the polarimetric study in Sect. 3.1 we pointed out that the wavelength location of the bump seems to be the same for both lines, which is not reproduced in the first three rows of this column. We have never detected this apparent mismatch between the location of the bump in both lines in the observations (see Fig. 1).

Finally, the fifth column shows a Gaussian perturbation whose center moves from the top to the bottom of the atmosphere. This LOS velocity stratification reproduces the displacement of the bump from the center of the line to the red wing, as the real profiles show. In this case the position of the bump seems to be almost the same for both lines, although it is sometimes poorly defined in the second line, Fe I $6302.5 \AA$. A Gaussian perturbation with a very large width will produce the same effect on the profiles as the LOS velocity model displayed in the third column owing to the narrow optical depth range of sensitivity for the two iron lines examined. This property implies that for the synthesis of the Stokes profiles a jump or a Gaussian with a large width will give the same results.

Because of the similarities between the real and synthetic profiles displayed in the sixth column of Fig. 4, we decided to employ the model with the Gaussian perturbation in the LOS velocity to invert the profiles. We used the SIRGAUSS inversion code (Bellot Rubio 2003) instead of SIR to obtain the atmosphere stratifications from the inversions of the Stokes profiles.

\section{Stokes profile inversions}

The SIRGAUSS code is a variation of the original SIR program that has as free parameters those corresponding to the background atmosphere together with the parameters needed to define a Gaussian perturbation for each physical quantity. The Gaussian perturbation profile is the same for all of the physical quantities, meaning that it has the same width and center, which allows one to set a different amplitude for each physical quantity.
The configuration for the inversion code is based on a twocomponent model with a nonmagnetic atmosphere and a magnetic atmosphere. The nonmagnetic atmosphere has freedom only in temperature and LOS velocity. The magnetic component uses a Gaussian perturbation in LOS velocity and temperature stratifications. The background under the Gaussian perturbation is kept constant to $0 \mathrm{~km} \mathrm{~s}^{-1}$ for the LOS velocity, but it is inverted for the temperature stratification. The magnetic field, the microturbulence, and the macroturbulence are constant with height, but their value can change. Inclination and azimuth angles are fixed to 0 degrees. Finally, the filling factor between components is also inverted during the process. As a result, the total number of free parameters is 12 .

\subsection{Monte Carlo analysis}

The complexity of the atmosphere implies that convergence to the most reliable solution is not granted. For this reason we made a statistical study to find the most probable solution that minimizes the $\chi^{2}$ parameter. We performed 100 inversions with the same initial atmosphere. In each inversion we added random noise to each Stokes parameter. The noise value was of the order of the real noise, $10^{-3} I_{\mathrm{c}}$, and is different for each Stokes parameter. From the results of the 100 inversions we obtained solutions that correspond to a different $\chi^{2}$ minimum. We selected the most common solution for the location of the center of the Gaussian perturbation by calculating the mode of the different results. Then we chose all the solutions that presented a Gaussian center position closer than \pm 0.1 (in $\log \tau$ units) to the most common perturbation center. The final solution of the inversion is the mean value of all of these selected inversion results. The atmosphere stratifications examined in the following sections were always obtained using this Monte Carlo simulation.

\subsection{Inversion results}

The results from the inversion of the Stokes profiles examined in Sect. 3 are shown in Fig. 5. Each row shows a different time for the same pixel, running from top to bottom, corresponding to the four different colors marked in Fig. 1. The first three columns show the atmospheric parameters of temperature, the magnetic field, and the LOS velocity. The last two columns show the observed and fitted Stokes $I$ and $V$ profiles.

The temperature shows a similar behavior for the four snapshots, being much hotter than the HSRA reference temperature (dashed line) during the process. The magnetic field intensity is strong, reaching values in the range $1-1.5 \mathrm{kG}$. It decreases slightly during the evolution of the bump through the center to the red wing of the line. The LOS velocity reproduces the same behavior as we showed in Fig. 4, a Gaussian perturbation that moves down into the photosphere from the top to the bottom layers. Note that the center position of the Gaussian also is a free parameter in the SIRGAUSS code. The amplitude of the Gaussian perturbation reveals velocity values higher than $6 \mathrm{~km} \mathrm{~s}^{-1}$, indicating that the plasma velocities involved in the process reach values higher than the estimated sound speed in the photosphere. Moreover, the Gaussian perturbation reaches deeper layers as the bump is moving through the profile until it reaches the red wing. The last two columns show that the fitted profiles obtained from the inversion (red lines) perfectly match the observed profiles (black lines). Finally, the value of the filling factor decreases with time in this particular pixel, although, as we show below, this is not the typical behavior. 

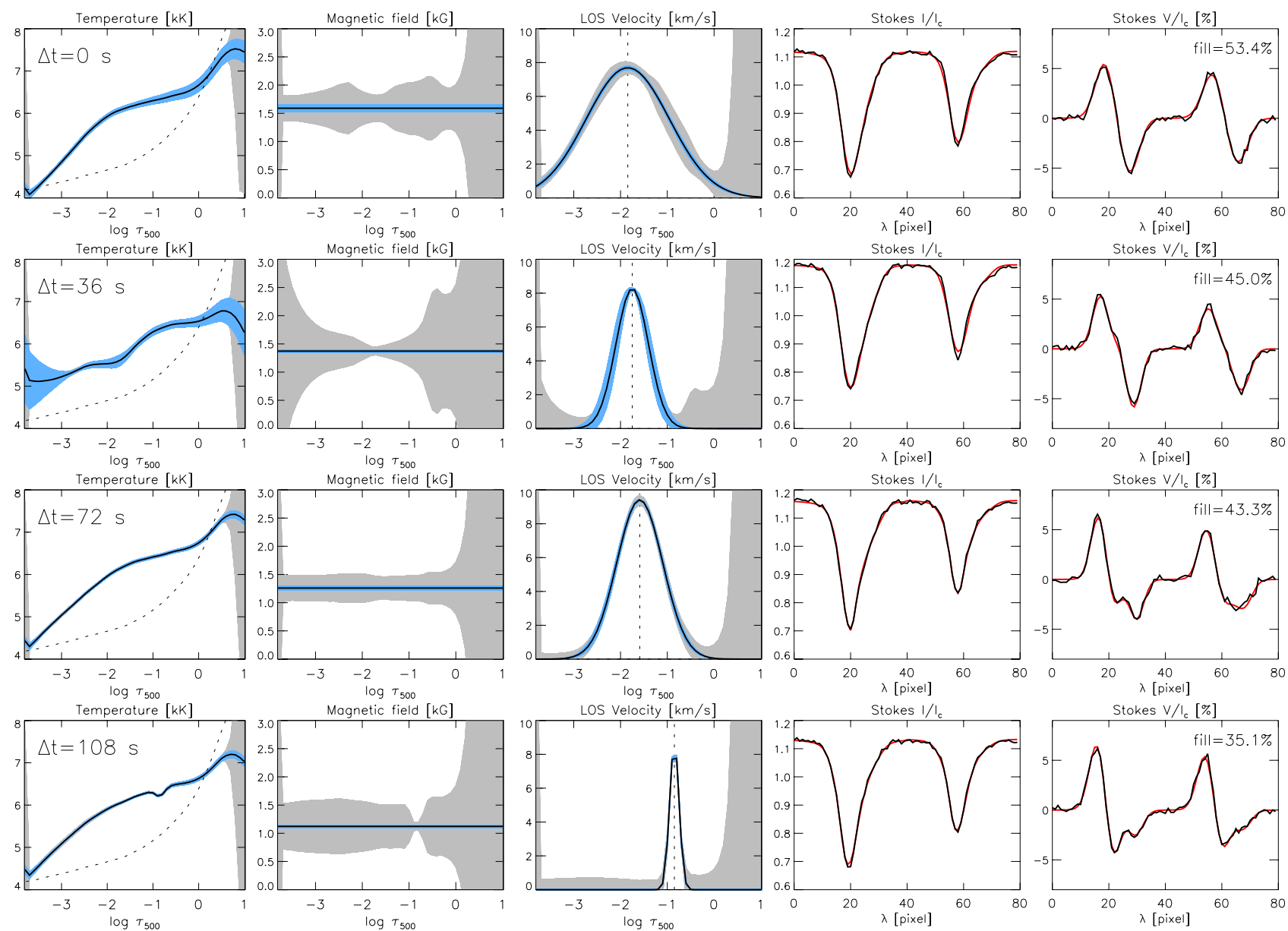

Fig. 5. Model atmosphere and Stokes profiles resulting from the SIRGAUSS inversion from one of the detected events. Each row corresponds to a different time running from top to bottom. The Stokes profiles are the same as presented by the color lines in Fig. 1. The first column shows the temperature stratification. Second and third columns are the magnetic field and the LOS velocity stratifications. The black line designates the mean atmosphere obtained from the Monte Carlo simulation, blue is the deviation from this mean solution, and gray represents the nonsensitive regions (large gray regions indicate that the response of the Stokes profiles to perturbations in the model is very weak). The last two columns show the observed Stokes $I$ and $V$ profiles in black and the fitted ones in red. The dashed line in the first column designates the HSRA reference atmosphere temperature, the vertical line in the third column marks the center of the Gaussian perturbation.

\section{Physical quantities outside hydrostatic equilibrium}

As a first approach, we performed the inversions assuming hydrostatic equilibrium (hereafter, HE). However, the results obtained in the previous section necessitated considering dynamical terms in the equation of motion because the LOS velocity stratification reaches values that are higher than the sound speed in the photosphere. The nonequilibrium nature of the atmosphere causes the HE gas pressure, gas density, and geometrical heights to be incorrect. However, the error that these physical quantities produce in the inverted Stokes profiles is negligible, which allows us to change these physical quantities by a few orders of magnitude with a consequent change in the Stokes profiles of about the noise level (Puschmann et al. 2010). Consequently, we do not need to repeat the inversion outside the HE conditions because we would obtain the same temperature, magnetic field, and LOS velocity stratifications. The aim of this section is to obtain the gas pressure, density and geometrical height stratifications compatible with the equation of motion, taking into account these high-velocity gradients. In addition, this equation of motion also uses the optical depth-dependent temperature and LOS velocity stratification deduced from the inversions.

\subsection{The Equation of motion}

To obtain a more accurate geometrical height scale where the Gaussian perturbation is moving down with time we need to solve the equation of motion (see for instance Priest 1984):

$\rho \frac{\mathrm{D} \boldsymbol{v}}{\mathrm{D} t}=-\nabla P+\boldsymbol{j} \times \boldsymbol{B}+\rho \boldsymbol{g}$,

with $\boldsymbol{j}$ and $\boldsymbol{g}$ the electrical current density vector and gravity. The remaining parameters are the gas density $\rho$ and gas pressure $P$, the magnetic field $\boldsymbol{B}$, and the velocity $\boldsymbol{v}$ vectors. In addition, the Lagrangian derivative can be defined by

$\frac{\mathrm{D}}{\mathrm{D} t}=\frac{\partial}{\partial t}+\boldsymbol{v} \cdot \boldsymbol{\nabla}$

We considered only the variations along the LOS and the $z$ axis aligned with the LOS direction. For this reason the variables are only height $(z)$ and time $(t)$ dependent. The $z$-component of the equation of motion can be written as

$\frac{\mathrm{d} P}{\mathrm{~d} z}+\rho g=-\rho\left(\frac{\partial v_{z}}{\partial t}+\frac{\partial \frac{1}{2} v_{z}^{2}}{\partial z}\right)$,

with $g=+274 \mathrm{~ms}^{-2}$. 
The term $\boldsymbol{j} \times \boldsymbol{B}$ is neglected because we assumed that the magnetic field is strictly vertical in the region where the event is detected. We cannot be sure of the accuracy of this assumption because the Stokes $Q$ and $U$ amplitudes are always lower than the noise. However, if the magnetic field is strong enough, its inclination should be small because otherwise we would have Stokes $Q$ and $U$ signals that are higher than the noise level. To prove this, we synthesized the Stokes profiles using the atmospheres obtained in Sect. 5.2 and we found an upper limit for the magnetic field inclination of 10-15 degrees. If the inclination values are higher than this limit, the Stokes $Q$ and $U$ signals would be clearly distinguishable from the noise signal. Consequently, owing to the high signals of the Stokes $V$ profiles and the small area covered by these features, the assumption of a vertical field concentrated inside an intergranular lane is probably reliable.

\subsection{Solution of the equation of motion}

We rewrote the equation of motion as a function of continuum optical depth at $500 \mathrm{~nm} \tau_{500}$ to find an expression that preserves the optical depth dependence of the temperature and LOS velocity stratifications assumed in the inversions. We write the ideal gas equation neglecting radiation as

$\rho=a P$,

where $a=\frac{\mu}{R T}$, and $\mu$ is the mean molecular weight. From the definition of optical depth we can write

$\tau_{500} \ln (10) \operatorname{dlog}_{10} \tau_{500}=-\kappa \rho \mathrm{d} z$,

where $\kappa$ is the continuum absorption coefficient at $500 \mathrm{~nm}$ per gram. We then define $x=\log _{10} \tau_{500}$ and, using Eq. (6), we obtain

$\tau_{500} \ln (10) \mathrm{d} x=-\kappa a P \mathrm{~d} z$.

We define a new variable $h$ as

$h=\frac{P \mathrm{~d} z}{\mathrm{~d} x}=-\frac{\tau_{500} \ln (10)}{\kappa a}$.

We assume that the two parameters $h$ and $a$ do not depend on the gas pressure, that is, they are only functions of the temperature. This is because the continuum absorption coefficient $\kappa$ only weakly depends on the gas pressure given by the Saha equation. We have checked that the variation of the continuum absorption coefficient with temperature $\left(\frac{\mathrm{d} \kappa}{\mathrm{d} T}\right)$ is many orders of magnitude stronger than the variation of the continuum absorption coefficient with the gas pressure $\left(\frac{\mathrm{d} \kappa}{\mathrm{d} P}\right)$. For this reason, we assume that the continuum absorption coefficient only depends on the optical depth, i.e. $\kappa=\kappa\left(\tau_{500}\right)$. On the other hand, we have calculated the compressibility coefficient $\alpha=\left(\frac{\partial \ln \rho}{\partial \ln \mathrm{P}}\right)_{T}$ and found that $\alpha \simeq 1$ throughout the deep layers. As, from Eq. (6), $\alpha=1+\left(\frac{\partial \ln a}{\partial \ln P}\right)_{T}$, we can assume that to first order $a=a(T)$. This result is produced by the low dependence of the mean molecular weight on gas pressure changes. In fact, a change of $1 \%$ in the mean molecular stratification is obtained when we change the gas pressure by two orders of magnitude. Then, given that the temperature stratification obtained from the inversions does not depend on the gas pressure, i.e. $T=T\left(\tau_{500}\right), h$ and $a$ are only function of the optical depth.

The next step is to introduce into Eq. (5) the definition of $d x$ from the first term of Eq. (9) and the definition of $a$ using Eq. (6). After some algebra we obtain

$\frac{\mathrm{d} P}{\mathrm{~d} x}+A(x)-P B(x)=0$,

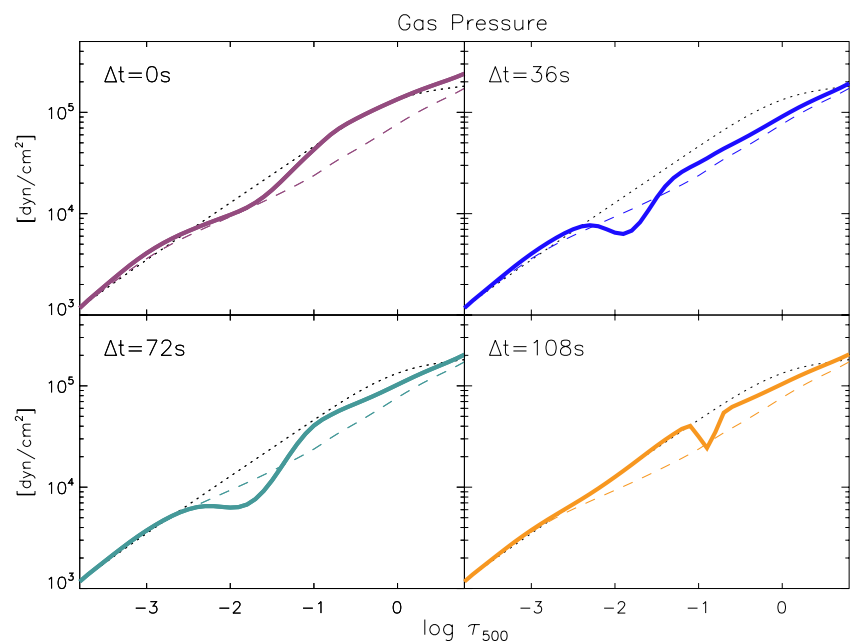

Fig. 6. Evolution of the gas pressure stratification outside HE assumption (solid). We maintain the color criteria used in Fig. 1, purple for $\Delta t=0$, blue for $\Delta t=36$, green for $\Delta t=72$ and orange for $\Delta t=108$. Dashed lines are the solutions under HE and the dotted line is the gas pressure for the HSRA reference model.

with

$A(x)=a h\left(g+\frac{\partial v_{z}}{\partial t}\right)$

$B(x)=-a\left(\frac{\mathrm{d}\left(\frac{1}{2} v_{z}^{2}\right)}{\mathrm{d} x}\right)$.

The solution of the linear differential Eq. (10) is the following:

$P(x)=\mathrm{e}^{\int_{x_{0}}^{x} B(x) \mathrm{d} x}\left[P_{0}-\int_{x_{0}}^{x} A(x) \mathrm{e}^{-\int_{x_{0}}^{x} B(x) \mathrm{d} x} \mathrm{~d} x\right]$.

To solve Eq. (13) we need to calculate the variation of the LOS velocity $\left(v_{z}\right)$ with time $\left(\frac{\partial v_{z}}{\partial t}\right)$. We deduced this variation from the four different LOS velocity stratifications presented in Fig. 5. We analyzed the temporal variation of the entire LOS velocity stratification at every instant, obtaining the derivative of $v_{z}$ for each optical depth point of the vector. This term has a high uncertainty because we can only use four points to calculate it; the bump is only seen in four different instants in the Stokes $V$ profile owing to the limited cadence of the observation.

\subsection{Gas pressure}

The results for the gas pressure stratification for the four different positions of the bump inside the Stokes $V$ profile, as marked by different colors in Fig. 1, are shown in the panels of Fig. 6. We have considered for the integration of this equation that $P_{0}$ (the gas pressure at $\log \tau_{500}=-3.8$ ) has the same value as shown by the HSRA reference atmosphere model at the top boundary. This is why both lines, the reference atmosphere (dotted) and the gas pressure outside the equilibrium (solid), are joined at the top of the atmosphere.

If we compare the results of the HE (dashed) and non HE (solid) assumptions, we find that the main difference is the Gaussian perturbation that appears in the nonequilibrium gas pressure stratification. This perturbation is placed at the same location as where the LOS velocity perturbation is; it also moves 


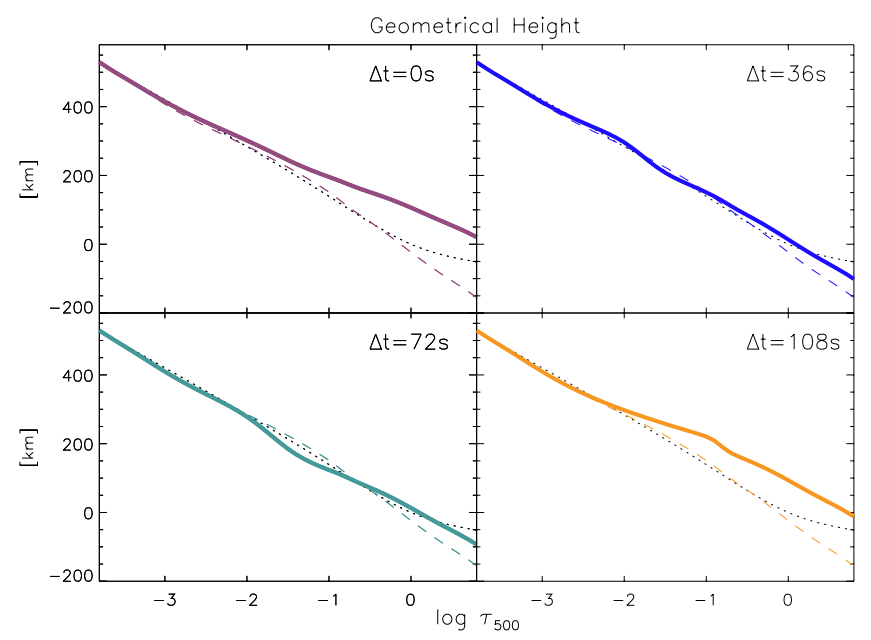

Fig. 7. Same as Fig. 6 for the relation between the optical depth and the geometrical height.

to deeper layers when the event evolves. Finally, the nonequilibrium gas pressure stratifications show almost the same behavior as the HSRA reference model at higher layers, while they show slightly lower values than the HSRA reference atmosphere at the bottom of the photosphere.

\subsection{Geometrical heights}

Provided that in first order $h$ and $a$ are independent of the gas pressure, we can use Eq. (9) to evaluate $h$ from the results of the inversions and to obtain the dependence of the geometrical height with optical depth using the gas pressure stratification. We do not have enough information to establish where the zero value of the geometrical heights is and we have chosen to put it at the top of the atmospheric stratification, at the same level as the HSRA reference model atmosphere. The geometrical height outside the HE assumption (see Fig. 7) strongly diverges from the equilibrium results, which indicates that there is a large deviation in the relation between optical depth and geometrical heights because of the high-speed magnetized plasma along the LOS. This result implies that for the same optical depths we are sampling higher heights in the photosphere, which is consistent with the fact that the perturbed models are less dense and more transparent than the unperturbed ones.

\subsection{Atmospheric parameters versus geometrical heights}

From the result of the previous section we can plot the atmospheric parameters inferred using the SIRGAUSS inversion versus geometrical height, obtained after taking into account the dynamical terms in the equation of motion. The results are shown in Fig. 8. It is particularly interesting to examine the gas pressure and density, represented in the last two columns. Both magnitudes show a conspicuous negative Gaussian perturbation, located at the same height as the velocity perturbation, and a smooth behavior for the rest of the atmosphere stratification. Finally, the Gaussian perturbation shows a downward movement, as we found when we plotted the LOS velocity versus optical depth in Fig. 5. In addition, it also shows a width reduction during its evolution as if it were compressed when it moves towards denser layers. However, this behavior is clearly visible in this example, but is not as evident for the remaining detected cases.
The total distance the center of the Gaussian has covered in $108 \mathrm{~s}$ is almost $200 \mathrm{~km}$, indicating that the velocity of the center of the perturbation is nearly $2 \mathrm{~km} \mathrm{~s}^{-1}$.

\subsection{Mass of the downward perturbation}

The gas density shown in Fig. 8 reveals an inverse Gaussian perturbation that moves downwards to deeper layers in the solar photosphere. We analyzed the mass value of the region where the inverse Gaussian perturbation is located to determine its changes during the process. To do this we used the following equation

$\partial m=S \int \partial \rho \mathrm{d} z$

where $\partial m$ is the mass inside the volume occupied by the Gaussian perturbation on the density stratification, $\mathrm{d} z$ is the step in geometrical height, and $S$ is the section occupied by the perturbation. This section $S$ can be obtained as the pixel area multiplied by the filling factor of the magnetic component, $S=A f$. The density $\partial \rho$ used to obtain the mass of the perturbation is the sum of all the density values from $-2 \sigma$ to $2 \sigma$ with respect to the Gaussian center, $\sigma$ being the width of the Gaussian. We plotted in Fig. 9 the results for the total mass for the same time evolution as is shown in colored lines in Fig. 1. To estimate the uncertainty of these mass values we calculated the mass value for every different solution selected from the Monte Carlo analysis. The results presented in Fig. 9 indicate a decrease in the mass as the perturbation moves to deeper layers. But if we take into account the large uncertainty of each point, the results might be compatible with a constant mass value during the whole process.

\subsection{Heat exchange}

The entropy variations of the perturbation were obtained from changes in temperature and gas density as in Cox (1968):

$\mathrm{d} q=T \mathrm{~d} s=c_{v} \mathrm{~d} T-\frac{P \delta}{\rho^{2} \alpha} \mathrm{d} \rho$,

where $c_{v}$ is the specific heat at constant volume, $\alpha$ is the compressibility coefficient defined as $\alpha=\left(\frac{\partial \ln \rho}{\partial \ln P}\right)_{T}$ and $\delta$ is the thermal expansion coefficient given by $\delta=-\left(\frac{\partial \ln \rho}{\partial \ln T}\right)_{T}$. The entropy and heat exchanges, $\mathrm{d} s$ and $\mathrm{d} q$, are defined per mass unit. To obtain the variation of the entropy of a mass element we introduced in Eq. (15) the Lagrangian variation of the gas density and temperature.

Figure 10 shows the result of the heat exchange for the four different time steps with the same color code as used in Fig. 1. The four lines show the same behavior, presenting a smooth shape at the top and at the bottom of the atmosphere, negative values at the bottom part of the perturbation, and low positive values at the top part of the perturbation. The explanation of this behavior could be that the first part of the perturbation moves to deeper layers and emits radiation into the surrounding plasma that produces the detected negative heat exchange. At the same time, the last part of the perturbation is cooler than the plasma that it leaves behind during its descent, so the heat exchange of the perturbation is positive because it absorbs radiation from the external plasma. In all of these steps, the behavior is the same although the intensity of the exchange heat is increasing during the process. Finally, the consequence of the significant departure from adiabaticity $(\mathrm{d} s=0)$ is that the radiative exchange of the perturbation with its surroundings has to be efficient and fast. 
C. Quintero Noda et al.: Photospheric downward plasma motions in the quiet Sun

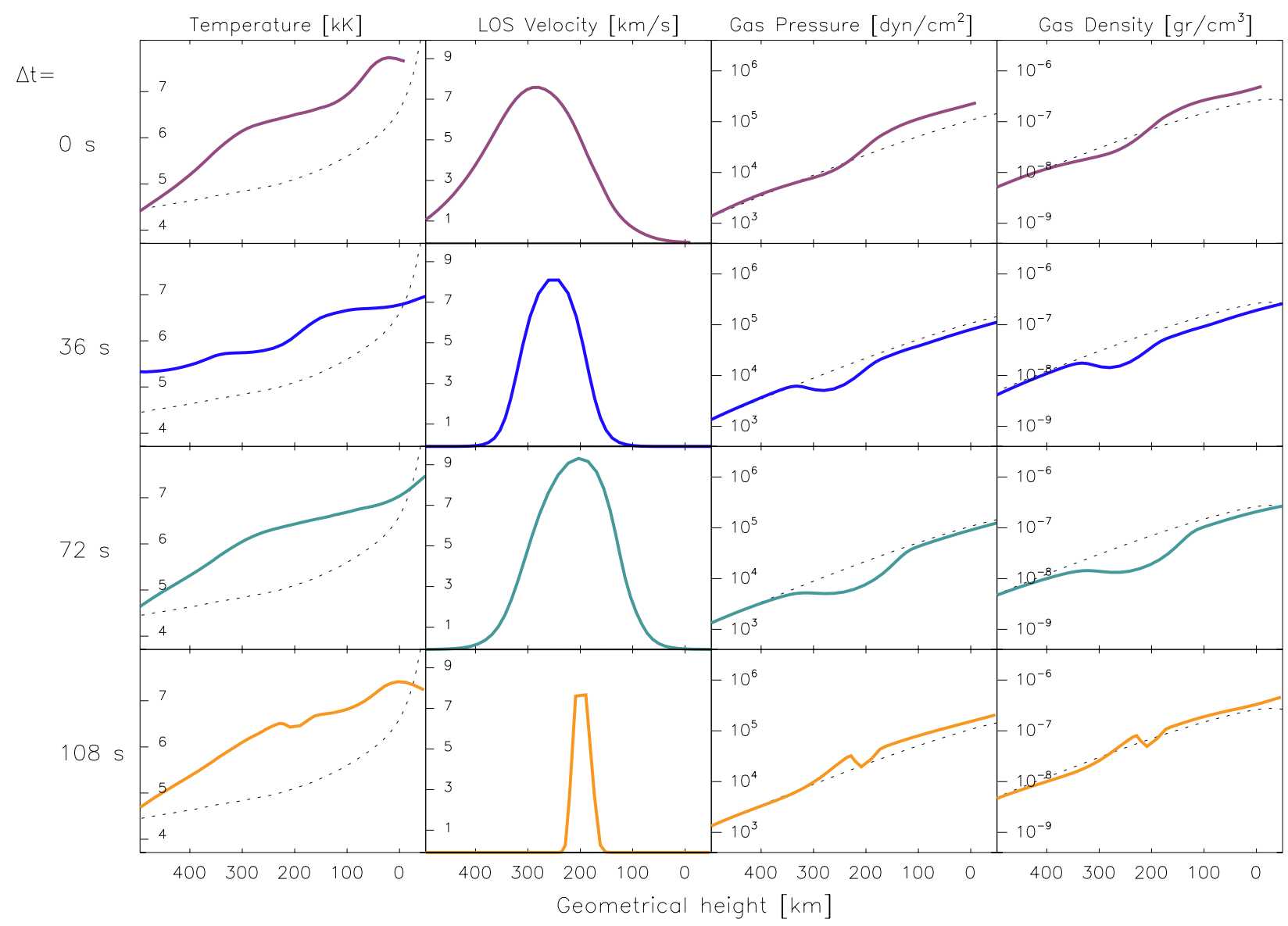

Fig. 8. Atmospheric parameters versus geometrical height. There are four columns with temperature, LOS velocity, gas pressure, and density depicted as solid lines. Dashed lines come from the HSRA reference model. The four rows correspond to the different time intervals defined in Fig. 1 with color lines. The geometrical heights from each row are different and come from those shown in Fig. 7.

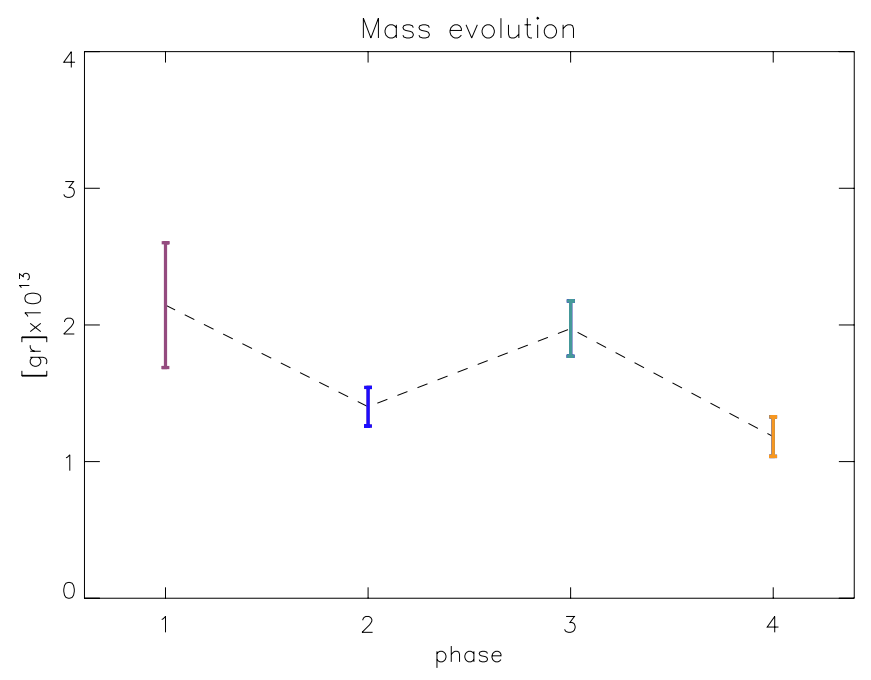

Fig. 9. Variation of the total mass during the event. There are four time steps that are the same as presented with colored lines in Fig. 1.

In the next section we obtain the radiative cooling time of the perturbation to determine whether this is feasible.

\subsection{Radiative cooling time}

To obtain an estimate of the time needed by a perturbation to exchange heat with its surroundings we followed the method used by Montesinos \& Thomas (1993) for the analysis of syphon

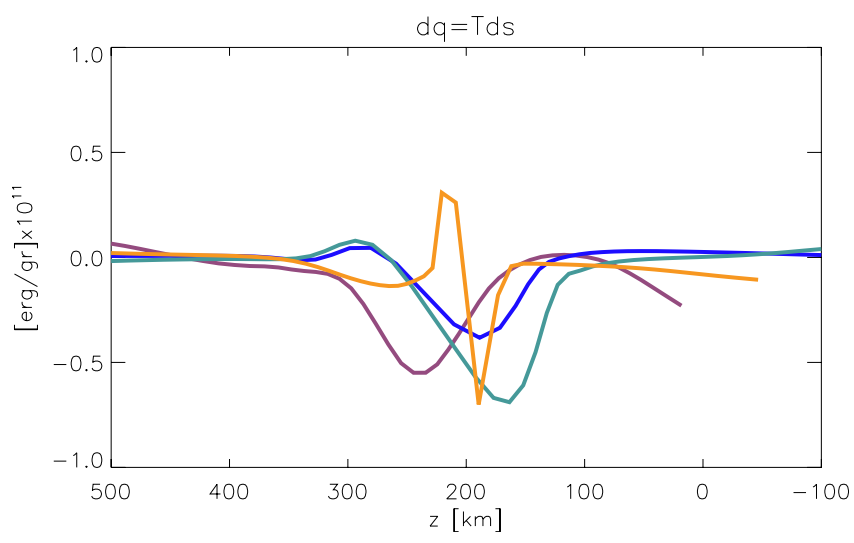

Fig. 10. Heat exchange that the perturbation undergoes during the process. We have used the same color code as in Fig. 1.

flows including radiative transfer. We assumed that the perturbation is within the optically thin limit. The radiative cooling time is then given by

$\tau_{\mathrm{rad}}=\frac{c_{p}}{16 \kappa \sigma T^{3}}$,

where $c_{p}$ is the specific heat at constant pressure, $\sigma$ is the Stephan-Boltzmann constant, and $\kappa$ the Rosseland opacity that can be approximated by

$\kappa=1.376 \times 10^{-23} p^{0.738} T^{5}$,

where the units are given in $\mathrm{cm}^{2} / \mathrm{gr}$. 


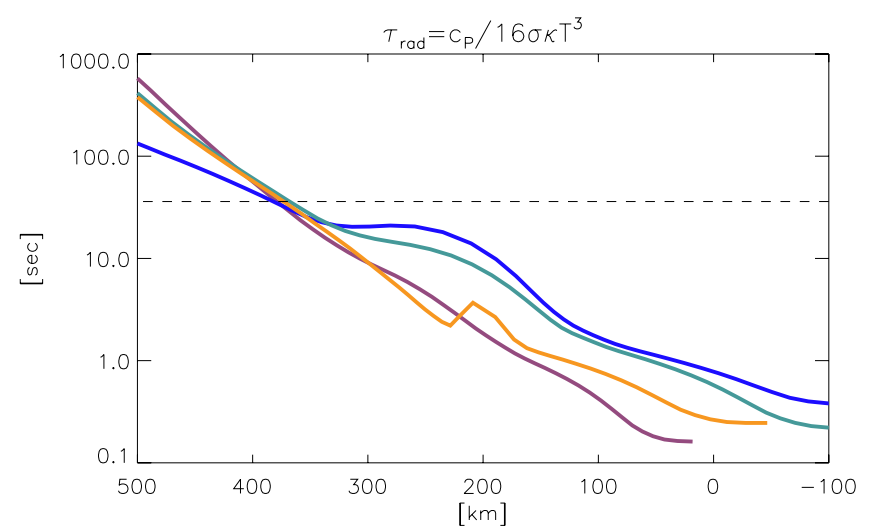

Fig. 11. Radiative cooling time for the Gaussian perturbation considering that it is optically thin. There are four colored lines with the same color code as used in Fig. 1. The horizontal line represents the time cadence of the observation.

Figure 11 shows the radiative cooling time for the four different locations of the bump inside the Stokes $V$ profile. The gas pressure and temperature stratification used to obtain the cooling time are the same as shown in Fig. 8. The horizontal line represents the time cadence of our observation, i.e. $36 \mathrm{~s}$. The trace of the Gaussian perturbation is also present in the time stratification as a local time increase. All of the lines have a time value below the time cadence of the observation, where the Gaussian perturbation signal is located. Consequently, the time needed by the perturbation to reach equilibrium is much shorter than the time between observations, which indicates that the perturbation is in thermal equilibrium with its surroundings.

\subsection{Total heat, work, and internal energy}

The work done by external forces over the mass element can be defined as

$\mathrm{d} w=\frac{P \delta}{\rho^{2} \alpha} \mathrm{d} \rho$,

and the total value of the work for every instant where the bump is visible inside the Stokes $V$ profile as

$$
\mathrm{d} W=\int(\mathrm{d} w) \mathrm{d} m=\int(\mathrm{d} w) S \partial \rho \mathrm{d} z
$$

where the section $S$, the increment in height $d z$, and the density perturbation $\partial \rho$ were described in Sect. 6.6.

To calculate the work we repeated the same steps as in Sect. 6.7 to obtain the heat. Then we obtained the total heat $\mathrm{d} Q=\int(\mathrm{d} q) \mathrm{d} m$ and the total work $d W$ by integrating these quantities inside the volume occupied by the mass element. Finally, we calculated an uncertainty value for the total heat and total work in each time step using the different Monte Carlo solutions, see Sects. 5.1 and 6.6.

The sum of both elements will give us the total internal energy of the perturbation. We show the evolution of these three thermodynamic parameters in Fig. 12. At the beginning, the mass element dissipates heat at a high rate, and when it decreases in the photosphere, the heat exchange with the external medium is reduced. The positive sign of the work means that the perturbation is compressed because of the encounter with denser layers while it is moving downwards. Finally, the internal energy, as a sum of heat and work, is constant, inside the error bars, and close to zero because the pressure increment due to the encounter with

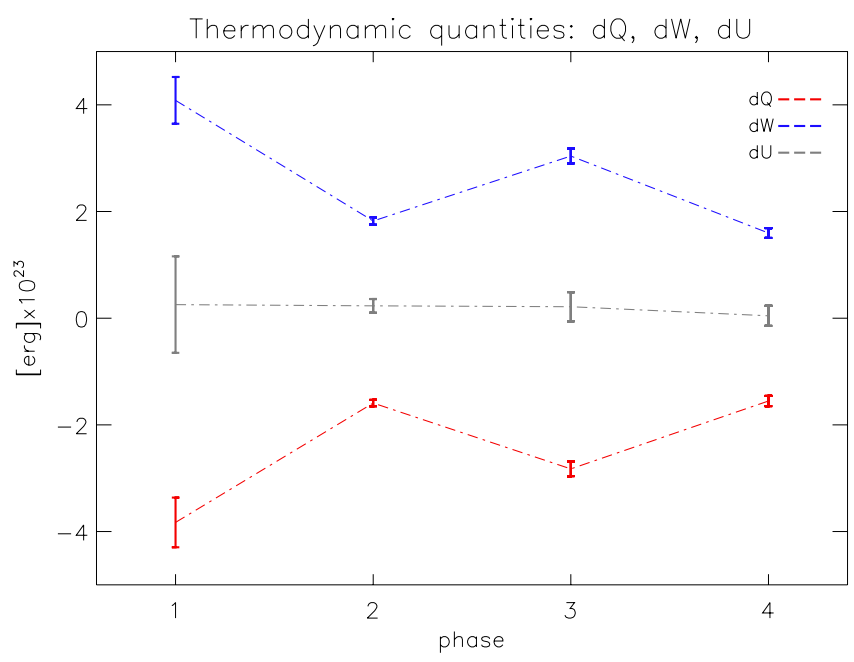

Fig. 12. Total heat, work, and internal energy. The heat is represented in red, the work is shown in blue, and the internal energy, as the sum of the two previous magnitudes, is represented in gray. There are four points that correspond to the different bump positions inside the Stokes $V$ profile, as Fig. 1 shows.

a denser medium is nearly compensated for the heat radiated by the perturbation. This behavior indicates that the evolution of the mass element occurs under nonadiabatic conditions; all the energy gained by the compression of the downward mass motion is effectively radiated.

\section{Analysis of many events}

We analyzed a single event in detail in the previous sections. In this section, we study many events to determine whether the properties found and described before are the same for the remaining cases. To carry out this study we selected pixels from the two normal Hinode/SP scan maps presented in Fig. 2. We chose the profiles inside the different redshifted patches placed over the whole map that show the same spectral properties and time steps. Then we inverted them using the same procedure as in Sects. 5 and 6. However, this time we performed the Monte Carlo analysis with only 20 inversions per profile. We also changed the method used to obtain the physical quantities outside the hydrostatic equilibrium hypothesis because we examined the Hinode/SP normal maps. We only used the variation of the atmospheric parameters with optical depth because we had no information on the temporal evolution. We rewrote Eq. 5 for this scenario as

$\frac{\mathrm{d} P}{\mathrm{~d} z}+\rho g=-\rho \frac{\partial \frac{1}{2} v_{z}^{2}}{\partial z}$.

If we assume that the magnitude of the redshift of the bump in the Stokes $V$ profile is directly related to the temporal evolution, we can search for pixels that correspond to the different phases represented in Fig. 1. We classified them by visual inspection and then separated the results from the analysis of each phase to facilitate visualization. We found 92 pixels for the first bump step ( $\Delta t=0$ in Fig. 1), 107 pixels for the second bump step $(\Delta t=36), 69$ profiles for the third bump step $(\Delta t=72)$, and 57 pixels for the last bump step $(\Delta t=108)$. Figure 13 shows the physical parameters obtained from these samples through the inversion of the Stokes profiles following the same method as presented in Sect. 5. We show the results for the different atmospheric parameters in the region where the center of the 
C. Quintero Noda et al.: Photospheric downward plasma motions in the quiet Sun
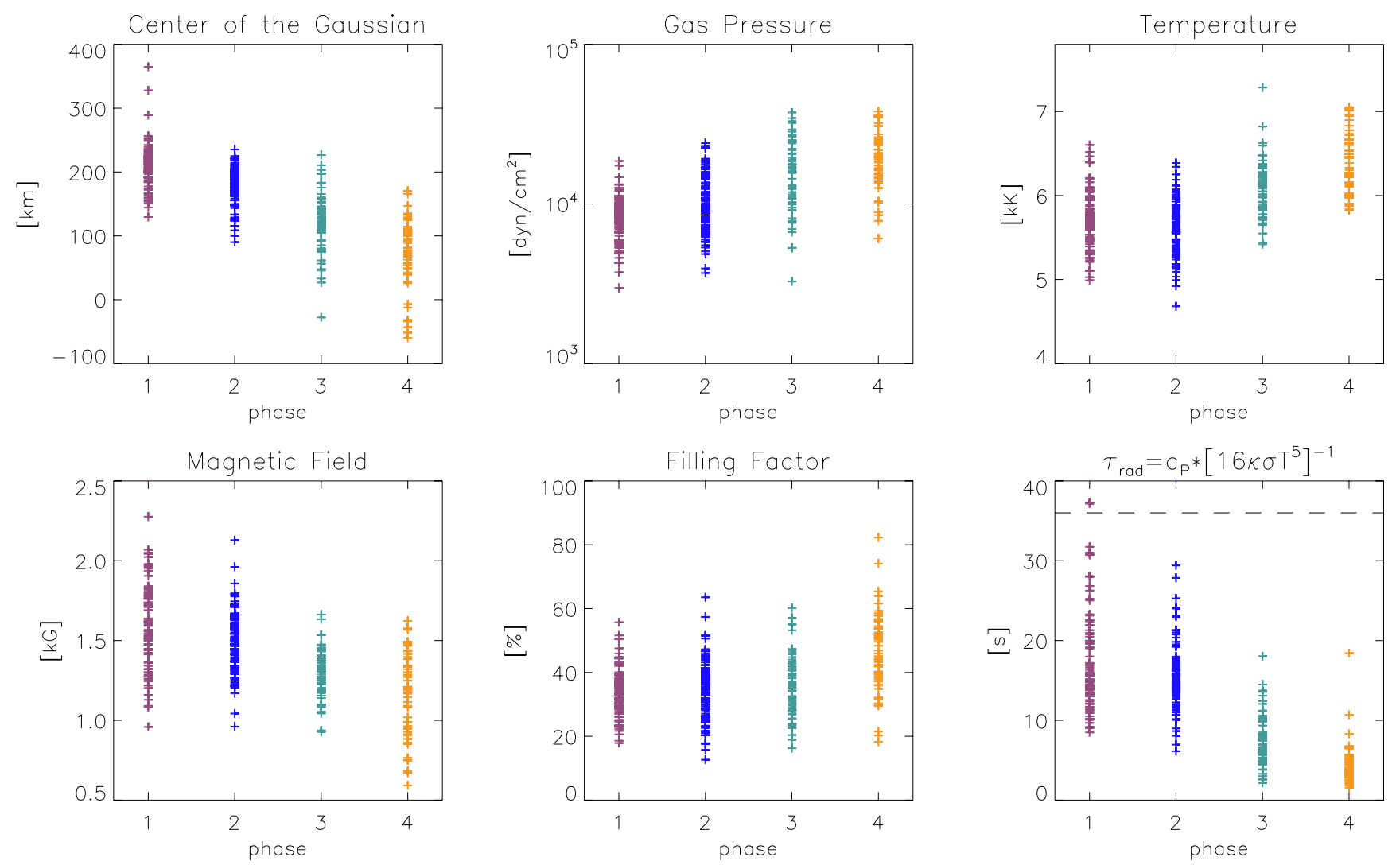

Fig. 13. Statistical results for the inversions of a large number of pixels selected from the maps presented in Fig. 2. We have used, in order, 92 , 107, 69 and 57 pixels for each different phase presented with different colored lines in Fig. 1. We have kept the same color code for each bump step. The first panel shows the location of the center of the Gaussian perturbation for each phase. The second top panel shows the gas pressure at the center of the Gaussian, and the third top panel displays the temperature at the same location. The first panel of the second row presents the value of the magnetic intensity for each phase. The second bottom panel shows the filling factor of the magnetic component, and the last panel displays the radiative cooling time for each phase.

Gaussian perturbation is located. The temperature increases during its evolution, while the perturbation moves downwards and enters the hotter plasma. This characteristic agrees with the total heat exchange presented in Fig. 12, where the heat exchange with the external medium decreases in the last steps because it is in equilibrium with the surrounding material. The first panel of the second row shows the slow decrease of the magnetic field intensity during the evolution of the perturbation. The following panel shows the slight increase of the magnetic filling factor with the evolution of the process. Because the magnetic field diminishes while the filling factor shows the opposite behavior, the resulting magnetic flux will be almost constant during the process. Finally, the last panel shows the radiative cooling time at the center of the perturbation. Its value is lower than the horizontal line that represents the cadence of the raster scan mode (36 s). This last property agrees with the results of the previous section that point to a nonadiabatic process.

In summary, the Gaussian perturbation descends to deeper layers while it emits heat to the surrounding area until it reaches an equilibrium state (see Fig. 12).

\section{Discussion}

The analysis of the distorted profiles detected by the Hinode/SP instrument points to a possible mechanism that would originate in higher layers, probably at the bottom of the chromosphere, because we found a correlation with $\mathrm{Ca}$ II $\mathrm{H}$ bright points. The process then evolves and descends, leaving a detectable signal in
Mg I $b_{2}$ Dopplergrams and, at the end, it reaches the low photosphere, producing the strong polarimetric signal we analyzed in the Fe I 6301.5 and $6302.5 \AA$ lines. However, after our careful and detailed analysis, remains unanswered the important question of which physical process produces these strong flows.

A possible mechanism that can produce strong downflows in the quiet-Sun photosphere is a syphon flow process, as studied by Rüedi et al. (1992) and Montesinos \& Thomas (1993). However, that mechanism seems inappropriate for explaining this process because we did not detect the other associated footpoint that would have showed an upward velocity or the linear polarization signal that this loop configuration would produce in its top part. We only observed a single polarity region with strong fields, before the appearance of the redshifted signal, during the process and after the disappearance of the peculiar Stokes $V$ profiles.

Another mechanism that might produce these features is a convective collapse process (Parker 1978; Webb \& Roberts 1978; Spruit 1979). Photospheric layers are places prone to harbor a convective collapse event because that is where the horizontal granular flows lead to the concentration of enough magnetic flux in the intergranular lanes (Schüssler 1990, and references therein) that would produce the convective instability. Shimizu et al. (2008) and Fischer et al. (2009) have suggested that the mechanism behind the downflow events they examined is a convective collapse. This mechanism implies an intensification of the magnetic field after the strong downflows, but we have found the opposite behavior: the magnetic field decreases 
during the process. Furthermore, the convective collapse process usually ends with the formation of a stable magnetic flux tube (Nagata et al. 2008), but our results do not seem compatible with this scenario, at least in the iron line formation region, because almost all the detected events vanish after the disappearance of the strongly redshifted signal. In addition, the destruction of the magnetic flux tube is sometimes related to a rebound of material that appears when the downward magnetized plasma meets denser layers. This rebound would produce an upward stream (Grossmann-Doerth et al. 1998) and a strongly blueshifted lobe in the Stokes $V$ profiles (Bellot Rubio et al. 2001; Socas-Navarro \& Manso Sainz 2005). We found no trace of such blueshifted signals when the events vanish.

Another possibility we examined is a magnetic reconnection (Parker 1963) between field lines in the upper photosphere that might produce opposite jets. One of them, descending, would correspond to the features presented in this work (that we detected in the Hinode/SP data) and the other, ascending, would produce the bright point in the $\mathrm{Ca}$ II $\mathrm{H}$ spectral band that we found. This hypothesis leads us to the physical mechanism behind the type II spicules. Realistic MHD simulations proposed by Martínez-Sykora et al. (2011) showed that the origin of spicules involves dissipation of magnetic energy in nonadiabatic conditions. The main properties of this process are a reduction of the magnetic field strength, a downward shock, and a heating in the atmosphere. We have detected traces of these properties through the results of the inversion code. In addition, McIntosh \& De Pontieu (2009) have observed type II spicules in single polarity regions, which implies that the old conception of mixed polarity regions in the photosphere to produce a magnetic field reconnection is not always necessary.

However, we have no information on the chromosphere, so we cannot presume that we have detected the disk center photospheric trace of the formation of spicules. But what we can do is compare with another chromospheric process: rapid blueshifted excursions (RBEs), first announced by Langangen et al. (2008) as the disk counterpart of type II spicules. They found a strongly blueshifted signal in the chromosphere from measuring the Ca II line with high velocities of $15-20 \mathrm{~km} \mathrm{~s}^{-1}$ and short lifetimes of about $45 \mathrm{~s}$. The authors concluded that the origin of the RBEs has to be some kind of magnetic reconnection caused by the high energy released during the event. If a magnetic reconnection occurs and produces a very vertical and narrow blueshifted jet of material that is detected in the chromospheric layers, it seems plausible that the jet counterpart, narrow downward material, can be identified as the strong downflows we have examined in this work. They are very vertical and narrow, with lifetimes of $108 \mathrm{~s}$ and photospheric velocities near $10 \mathrm{~km} \mathrm{~s}^{-1}$. The RBEs are characterized to be detected in regions close to strong magnetic fields, but not on top of them, as we have found for the redshifted events, see Fig. 2. At this point, with all of these similarities between the two processes, we proposed that these strong downflows are the photospheric counterpart of the chromospheric RBEs, that is, of type II spicules.

\section{Conclusions}

We have carefully examined the quiet-Sun strong downflows detected with Hinode/SP as a redshifted Stokes $V$ signal far from the zero-crossing point. These events are located in intergranular lanes and appeared where the magnetic field is strong. There is no evidence of opposite polarity regions or linear polarization signal patches close to them. After examining the time series, we established a lifetime of $360 \pm 74 \mathrm{~s}$ and a mean size of $4 \pm 1.4$ pixels. The analysis of the normal maps revealed a rate of occurrence of $6 \times 10^{-3}$ cases per $\operatorname{arcsec}^{2}$.

The Stokes profiles present a characteristic polarimetric signal: Stokes $I$ shows a higher continuum signal and line core values than a quiet-Sun profile from an intergranular lane region. Stokes $V$ profiles show a bump that in the first moment is present in the upper part of the line at the zero-crossing wavelength. This profile evolves, presenting the same bump at different wavelength positions inside the line, in the red lobe and then in the red wing. According to the positions of the bump, the process evolution was classified into four different steps. The lifetime of each step is lower than the cadence of the time series, that is, $36 \mathrm{~s}$, because we always found a different step inside the pixel in consecutive images. The evolution of the wavelength position is always in the same direction, from the zero-crossing point of the line to the red wing. We found no bump in the blue lobe of the Stokes $V$ profiles.

The strong Doppler-shifted signal detected in the lower photosphere using Hinode/SP data was examined in higher atmospheric layers with the upper photospheric $\mathrm{Mg} \mathrm{I}_{2}$ line and the lower chromospheric $\mathrm{Ca}$ II $\mathrm{H}$ line. The $\mathrm{Mg}$ I $\mathrm{b}_{2}$ Dopplergrams presented a patch of strongly redshifted signal in the same place where the event is detected with the iron lines. At the same time, the $\mathrm{Ca}$ II $\mathrm{H}$ spectral band images displayed a bright point in the same region. Both the $\mathrm{Mg} \mathrm{I} \mathrm{b}_{2}$ and $\mathrm{Ca}$ II $\mathrm{H}$ images showed the associated pattern before, or at the same time as, the detection of the strong downflows in the Hinode/SP observation data, but never after the detection of the process by the photospheric iron lines.

The qualitative behavior of Stokes $V$ profiles was reproduced with models with a step in the LOS velocity stratification. To reproduce the Stokes $V$ amplitudes with the Fe I $6301.5 \AA$ line more intense than the Fe I $6302.5 \AA$ line, we chose a hotter atmospheric model than that of the HSRA reference atmosphere. The bump evolution on the Stokes $V$ profiles was obtained using a Gaussian perturbation in the LOS velocity that moves from the top to the bottom of the photosphere.

The atmospheric model compatible with the observed Stokes profiles was inferred using the SIRGAUSS inversion code. The model sequence revealed a Gaussian perturbation in the LOS velocity that moved downwards in the photosphere and was embedded in a hot atmosphere. The magnetic field intensity was higher than $\mathrm{kG}$ values during the whole evolution and slightly decreased during the different phases.

The departure from hydrostatic equilibrium in the models is strong because of the high velocities found, which are of about $10 \mathrm{~km} \mathrm{~s}^{-1}$. We obtained the gas pressure stratification taking into account the dynamical terms, $\frac{D v}{D t}=\frac{\partial v}{\partial t}+v \cdot \nabla v$, in the equation of motion. The perturbation in the LOS velocity stratification produces a negative Gaussian perturbation in the gas pressure and density at the same region where the LOS velocity shows it.

The analysis of the perturbation heat exchange with its surroundings showed that the front part of the event emits heat to the outside medium, which is cooler than the perturbation, while the rear part absorbs heat from the external media, which are hotter than the perturbation. This leads to a negative heat exchange in the front part of the perturbation and a positive heat exchange in the rear part. Assuming that the perturbation is optically thin, we employed the radiative cooling time definition used in the work of Montesinos \& Thomas (1993) to derive how long the perturbation needs to reach an equilibrium state with its surroundings. We obtained a time value lower than the time cadence of the 
observations, which points to a strongly nonadiabatic process. We also studied the evolution of the mass at the location of the perturbation and found that it is nearly constant, within the error bar uncertainty.

If we assume that the magnitude of the redshift of the bump in the Stokes $V$ profile is directly related to the temporal evolution, we can assume that the results of the study of the large number of cases found in the Hinode/SP normal map are compatible with the conclusions inferred from the time series observation. We found that the center of the Gaussian perturbation always moves downwards into the photosphere with time, while gas pressure and temperature at the center of the Gaussian perturbation increase with the evolution of the process. The magnetic field, chosen to be constant with height, decreases with the evolution of the perturbation, while the magnetic filling factor slightly increases, which keeps the magnetic flux value almost constant. Finally, the radiative cooling time is always much lower than the lifetime of the event, which confirms that it is a strongly nonadiabatic process.

The temporal variation of the perturbation displayed by the bump in the Stokes $V$ profiles, found in many cases, is compatible with a plasmoid of hot plasma that moves downward with high velocity. The different observed phases or steps correspond to the different optical depth locations of this plasmoid during its descent. This perturbation descends in nonadiabatic conditions, undergoes a compression by the denser external medium, and effectively radiates the heat generated by this compression. This behavior causes the perturbation to remain always in thermal equilibrium (nearly constant internal energy). The magnetic flux confined inside the perturbation also shows a constant value during the process because the decrease in the magnetic field intensity is compensated for the increase in the filling factor that can be understood as an increase in the horizontal size of the perturbation.

All these results support the idea of a new process that takes place at the bottom of the chromosphere and evolves as a downward motion until the mid-photosphere. To our knowledge, this is the first time that the evolution of this event is described through a detailed analysis of the Stokes profiles. The most probable mechanism is magnetic reconnection that takes place at the top of the photosphere or the bottom of the chromosphere. In this sense, this process might be related to the formation of type II spicules. So far, we have no polarimetric information on the chromosphere. For this reason, we need to wait for possible simultaneous polarimetric observing modes of the chromosphere and the photosphere that the future major projects such as EST, ATST and the space mission Solar-C will provide to reveal the nature of these highly dynamical events. It will also be necessary to examine realistic MHD simulations that cover the chromosphere and the photosphere to search for similar events and to investigate the mechanism that produces them.
Acknowledgements. This work has been funded by the Spanish MINECO through Projects No. AYA2009-14105-C06-03, AYA2011-29833-C06-03 and AYA2012-39636-C06-06. The data used here were acquired within the framework of Hinode Operation Plan 14 (Hinode-Canary Islands joint campaign). Hinode is a Japanese mission developed and launched by ISAS/JAXA, with NAOJ as a domestic partner, and NASA and STFC (UK) as international partners. It is operated by these agencies in cooperation with ESA and NSC (Norway).

\section{References}

Barthol, P., Gandorfer, A., Solanki, S. K., et al. 2011, Sol. Phys., 268, 1 Beckers, J. M. 1968, Sol. Phys., 3, 367

Bellot Rubio, L. R. 2003, in Solar Polarization, eds. J. Trujillo-Bueno, \& J. Sanchez Almeida, ASP Conf. Ser., 307, 301

Bellot Rubio, L. R., Rodríguez Hidalgo, I., Collados, M., Khomenko, E., \& Ruiz Cobo, B. 2001, ApJ, 560, 1010

Borrero, J. M., Martínez Pillet, V., Schlichenmaier, R., et al. 2010, ApJ, 723, L144

Borrero, J. M., Pillet, V. M., Schlichenmaier, R., et al. 2012, in 4th Hinode Science Meeting: Unsolved Problems and Recent Insights, eds. L. Bellot Rubio, F. Reale, \& M. Carlsson, ASP Conf. Ser., 455, 155

Borrero, J. M., Martínez Pillet, V., Schmidt, W., et al. 2013, ApJ, 768, 69

Cox, J. P. 1968, Principles of stellar structure - Vol. 1: Physical principles; Vol. 2: Applications to stars (New York: Gordon and Breach)

de Pontieu, B., McIntosh, S., Hansteen, V. H., et al. 2007, PASJ, 59, 655

Fischer, C. E., de Wijn, A. G., Centeno, R., Lites, B. W., \& Keller, C. U. 2009, A\&A, 504, 583

Gingerich, O., Noyes, R. W., Kalkofen, W., \& Cuny, Y. 1971, Sol. Phys., 18, 347

Grossmann-Doerth, U., Schuessler, M., \& Steiner, O. 1998, A\&A, 337, 928

Kosugi, T., Matsuzaki, K., Sakao, T., et al. 2007, Sol. Phys., 243, 3

Langangen, Ø., De Pontieu, B., Carlsson, M., et al. 2008, ApJ, 679, L167

Lites, B. W., Akin, D. L., Card, G., et al. 2013, Sol. Phys., 283, 579

Martínez Pillet, V., Del Toro Iniesta, J. C., Álvarez-Herrero, A., et al. 2011a, Sol. Phys., 268, 57

Martínez Pillet, V., Del Toro Iniesta, J. C., \& Quintero Noda, C. 2011b, A\&A, 530, A111

Martínez-Sykora, J., Hansteen, V., \& Moreno-Insertis, F. 2011, ApJ, 736, 9

McIntosh, S. W., \& De Pontieu, B. 2009, ApJ, 706, L80

Montesinos, B., \& Thomas, J. H. 1993, ApJ, 402, 314

Nagata, S., Tsuneta, S., Suematsu, Y., et al. 2008, ApJ, 677, L145

Parker, E. N. 1963, ApJS, 8, 177

Parker, E. N. 1978, ApJ, 221, 368

Priest, E. R. 1984, Solar magneto-hydrodynamics (Dordrecht: Reidel)

Puschmann, K. G., Ruiz Cobo, B., \& Martínez Pillet, V. 2010, ApJ, 720, 1417

Quintero Noda, C., Martínez Pillet, V., Borrero, J. M., \& Solanki, S. K. 2013, A\&A, 558, A30

Rüedi, I., Solanki, S. K., \& Rabin, D. 1992, A\&A, 261, L21

Ruiz Cobo, B., \& del Toro Iniesta, J. C. 1992, ApJ, 398, 375

Scharmer, G. B., Bjelksjo, K., Korhonen, T. K., Lindberg, B., \& Petterson, B. 2003, in SPIE Conf. Ser. 4853, eds. S. L. Keil, \& S. V. Avakyan, 341

Schüssler, M. 1990, in Solar Photosphere: Structure, Convection, and Magnetic Fields, ed. J. O. Stenflo, IAU Symp., 138, 161

Sekse, D. H., Rouppe van der Voort, L., \& De Pontieu, B. 2012, ApJ, 752, 108

Sekse, D. H., Rouppe van der Voort, L., \& De Pontieu, B. 2013, ApJ, 764, 164

Shimizu, T., Lites, B. W., Katsukawa, Y., et al. 2008, ApJ, 680, 1467

Socas-Navarro, H., \& Manso Sainz, R. 2005, ApJ, 620, L71

Solanki, S. K., Barthol, P., Danilovic, S., et al. 2010, ApJ, 723, L127

Spruit, H. C. 1979, Sol. Phys., 61, 363

Tsuneta, S., Ichimoto, K., Katsukawa, Y., et al. 2008, Sol. Phys., 249, 167

Webb, A. R., \& Roberts, B. 1978, Sol. Phys., 59, 249

Page 16 is available in the electronic edition of the journal at http://www . aanda.org 


\section{Appendix A: Fe I 5250.2 Stokes profiles}

Inspired by the results of Borrero et al. (2010), who first analyzed the quiet-Sun jets detected by Sunrise/IMaX and the possible detection of similar events by the later work of Martínez Pillet et al. (2011b), we synthesized the $5250 \AA$ spectral region (including the IMaX Fe I line and the neighboring Fe I $5250.6 \AA$ line) using the atmosphere obtained in Sect. 5.

Figure A.1 shows the results of this synthesis. We only displayed Stokes $I$ and $V$ because the inclination angle was fixed to zero degrees during the inversion process. Because of the high sensitivity of the Fe I 5250.2 A line to the temperature, the Stokes $I$ parameter is very weak for these models. The second line, Fe I $5250.6 \AA$, is less sensitive to the temperature and has a well-defined shape with the red wing strongly bent because of the high velocity gradients inside the atmosphere. The Fe I $5250.6 \AA$ line has a higher line formation region and is less sensitive to the magnetic field. However, similarly to the
Fe I 6301.5 and $6302.5 \AA$ lines measured by Hinode/SP, the Stokes $V$ amplitude signal is higher and the bump is more prominent in the line with the higher formation region. The bump has almost vanished in the line employed by the IMaX instrument, Fe $5250.2 \AA$.

We assumed that these events, because of the steep temperature gradients and the large heights of formation in the photosphere, were not detected by the line used by IMaX, even without the degradation effect produced by the spectral PSF of the instrument. The bump inside the profile of the second line has almost vanished, although in the last two steps it is still visible. The IMaX instrument would able to detect these events if it were measuring the second line. For this reason we have to assume that the events analyzed by Borrero et al. (2010) and the subsequent studies (Borrero et al. 2012, 2013; Quintero Noda et al. 2013) examined a different physical process. In fact, they established that most of the cases were upflow events and were related with linear polarization patches.

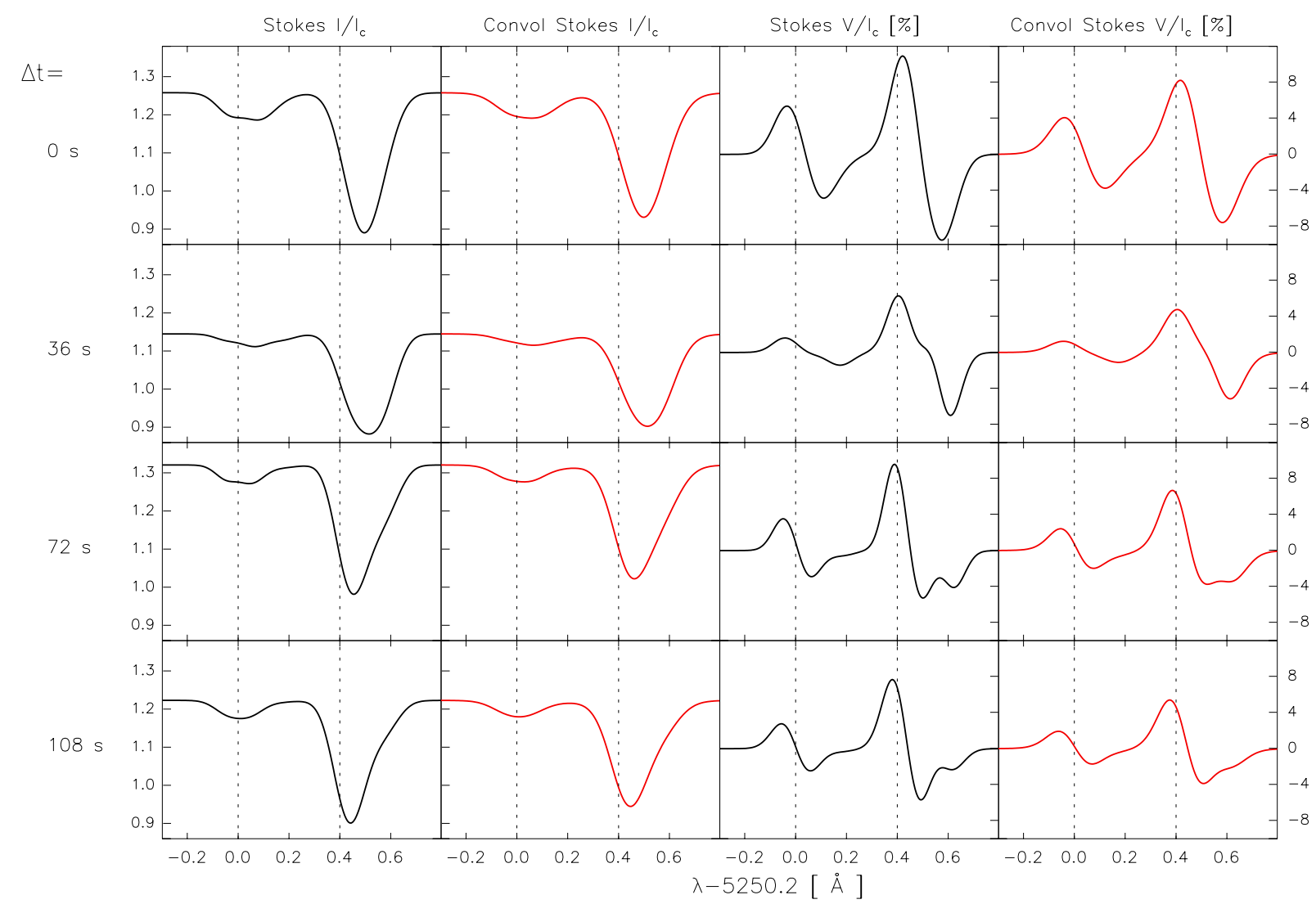

Fig. A.1. Synthetic Stokes $I$ and $V$ profiles for the Fe I 5250.2 and $5250.6 \AA$ lines. We have used the atmospheres presented in Fig. 5. Black lines are the original synthetic profile, while the red lines are the profiles after degrading them to the spectral resolution of IMaX. The dotted line designates the rest center of each line. 